\title{
Results and Perspectives of RHIC experiments
}

Timothy Hallmann ${ }^{\mathrm{a}}$

Brookhaven National Laboratory, Upton, NY 11973, USA

\begin{abstract}
We discuss Results and Perspectives of RHIC experiments beginning from an overview of the physics and experimental tools at RHIC and then introducing the highlights of the experimental results to date: (i) collective flow, (ii) hard probes and (iii) other supporting evidence for the creation of a strongly coupled quark-guon plasma. We give an outlook to further, new physics results to be expected from RHIC experiments.
\end{abstract}

\section{References}

1. M. Harrison, T. Ludlam and S. Ozaki (Eds.), 'The Relativistic Heavy Ion Collider Project: RHIC and its Detectors", Nuclear Instruments and Methods in Physics Research A 499, 235 (2003).

2. M. Gyulassy, L. McLerran, "New Forms of QCD Matter Discovered at RHIC', Nucl. Phys. A 750, 30 (2005).

3. J. Adams et al. (STAR collaboration), "Experimental and theoretical challenges in the search for the quark-gluon plasma: The STAR Collaboration's critical assessment of the evidence from RHIC collisions", Nucl. Phys. A 757, 102 (2005).

4. K. Adcox et al. (PHENIX collaboration), "Formation of dense partonic matter in relativistic nucleusnucleus collisions at RHIC: Experimental evaluation by the PHENIX Collaboration”, Nucl. Phys. A 757, 184 (2005).

5. I. Arsene et al. (BRAHMS collaboration), "Quarkgluon plasma and color glass condensate at RHIC? The perspective from the BRAHMS experiment", Nucl. Phys. A 757, 1 (2005).

6. B.B. Back et al. (PHOBOS collaboration), "The PHOBOS perspective on discoveries at RHIC', Nucl. Phys. A 757, 28 (2005).

\footnotetext{
a e-mail: hallman@bnl.gov
} 


\title{
Results and Perspectives of RHIC Experiments
}

\author{
T. Hallman \\ Dense Matter in Heavy lon Collisions and Astrophysics \\ Heavy Ion Summer School \\ July 14-26, JINR \\ Dubna, Russia
}

\section{Plan of this lecture}

- Overview of physics and experimental tools at RHIC

- Experimental results to date

- Collective Flow

- Hard Probes

- Additional supporting evidence

- New physics coming in the future

- Conclusions 


\section{The Science of RHIC}

- $\underline{\text { RHIC's original science mission: }}$

- Discovery of a new state of matter (quark-gluon plasma) in central heavy ion collisions $(\checkmark)$

- Detailed unfolding of the spin structure of the nucleon

- "Value added" physics:

- Low x structure of hadrons

- Fundamental tests of QCD

- Search for new exotics

\section{Forward inclusive spectra and correlations \\ Tagged forward proton studies \\ Ultra-peripheral collisions}

July 14-26 Dubna, Russia

The inspiration for the RHIC voyage of discovery: belief that under the right conditions, it is possible to "melt" protons and neutrons into their constituents

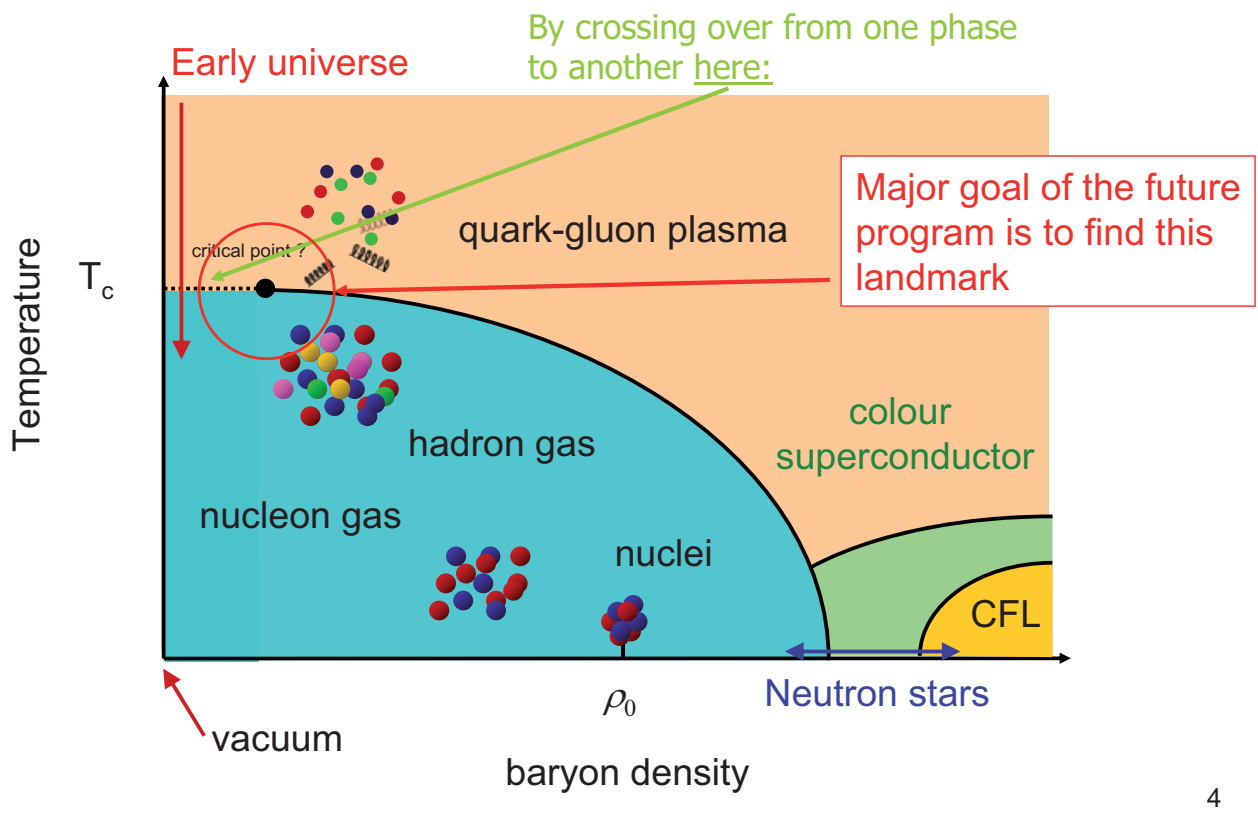


What are the phases of QCD Matter? What we expected: lattice QCD at finite temperature

\begin{tabular}{|l|}
\hline \multicolumn{1}{|c|}{ The nature of the transition? } \\
The most realistic lattice calculations suggest there are \\
no discontinuities in thermodynamic properties for the \\
conditions at RHIC (i.e., no 1 st. or $2^{\text {nd_order phase }}$ \\
transition), but that there is a smooth crossover \\
transition with a rapid evolution vs. temperature near \\
$T_{c} \approx 160-170 \mathrm{MeV}$
\end{tabular}

Critical energy density: $\varepsilon_{C}=(6 \pm 2) T_{C}^{4}$

$$
\mathrm{T}_{\mathrm{C}} \sim 175 \mathrm{MeV} \Rightarrow \varepsilon_{C} \sim 1 \mathrm{GeV} / \mathrm{fm}^{3}
$$

\section{Relativistic Heavy lon Collider}

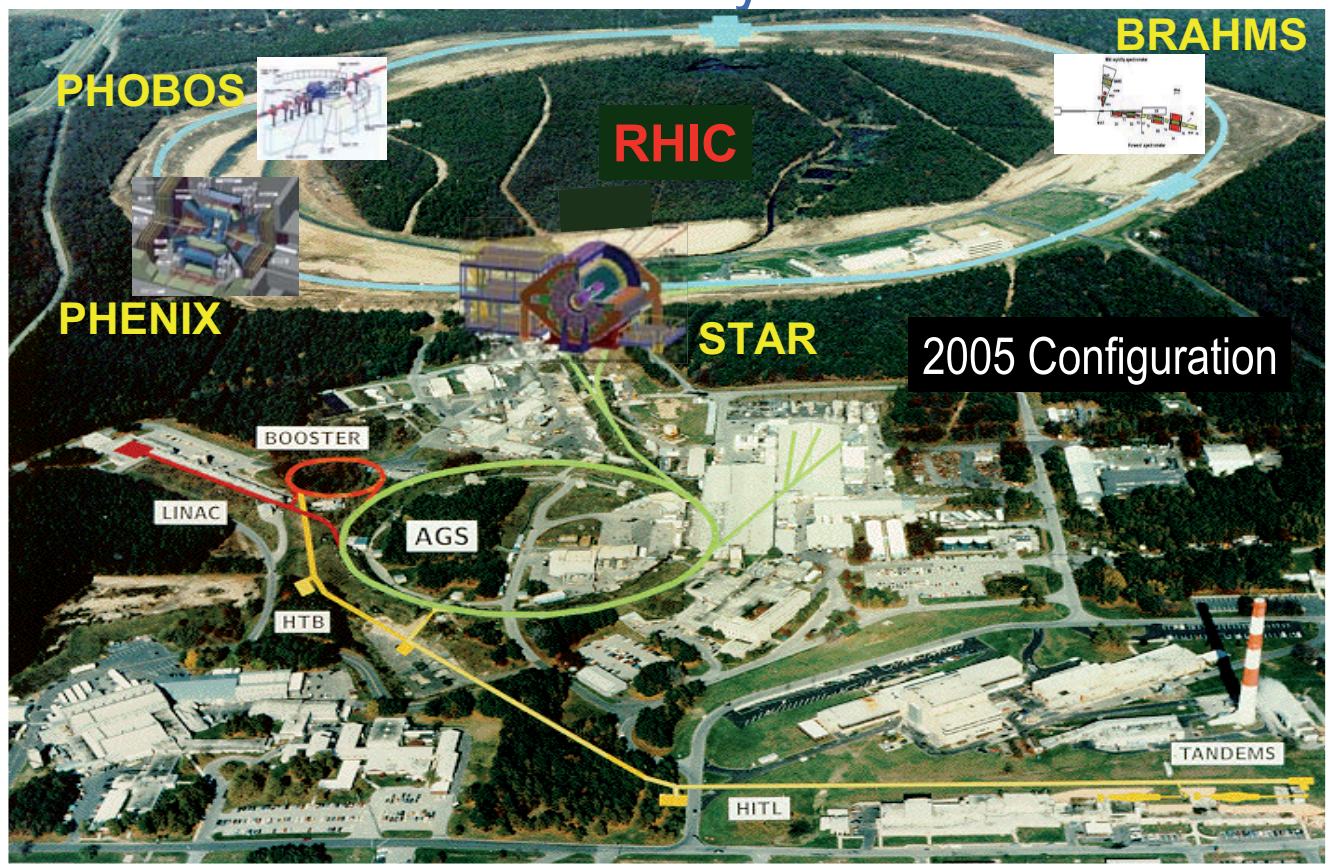




\section{Relativistic Heavy lon Collider}

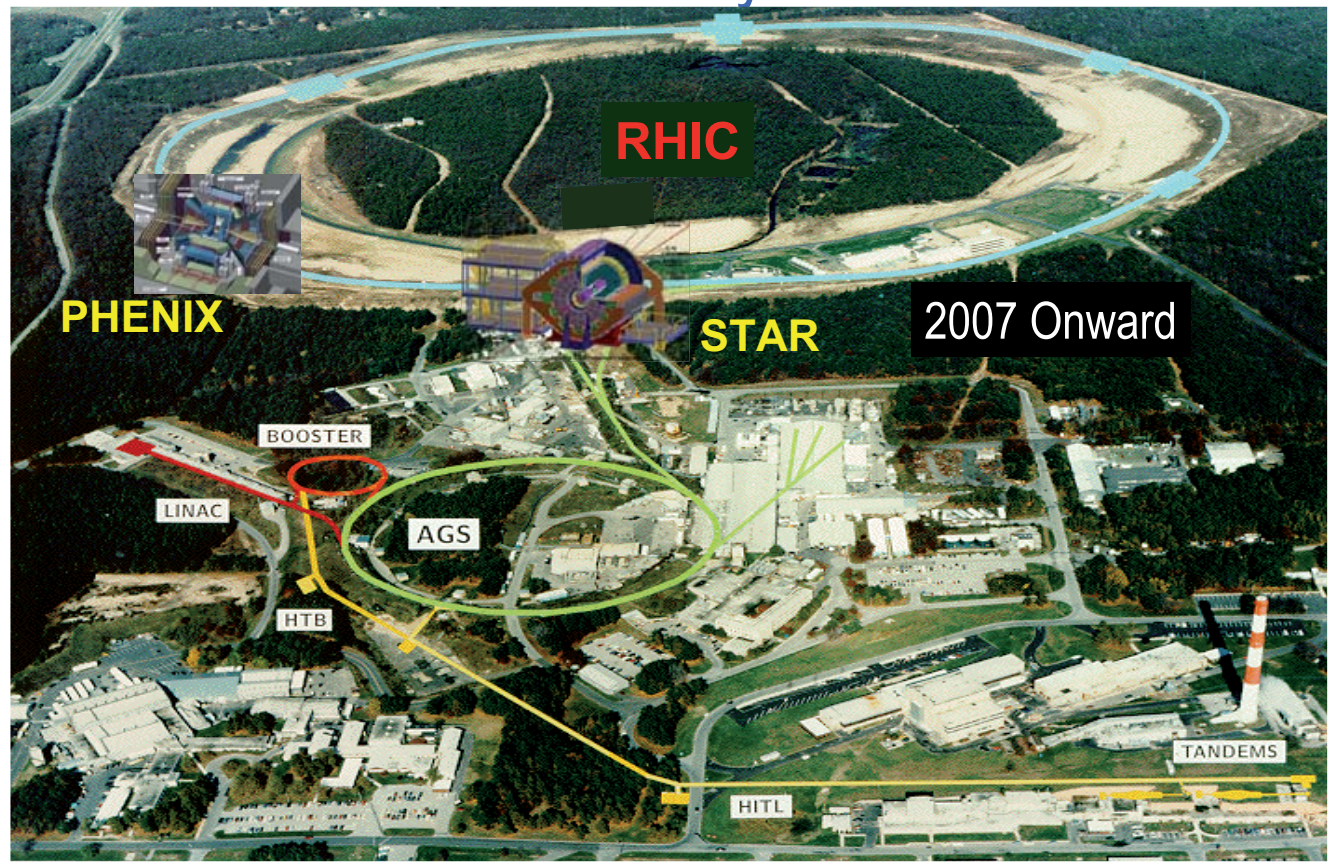

\section{Relativistic Heavy lon Collider}

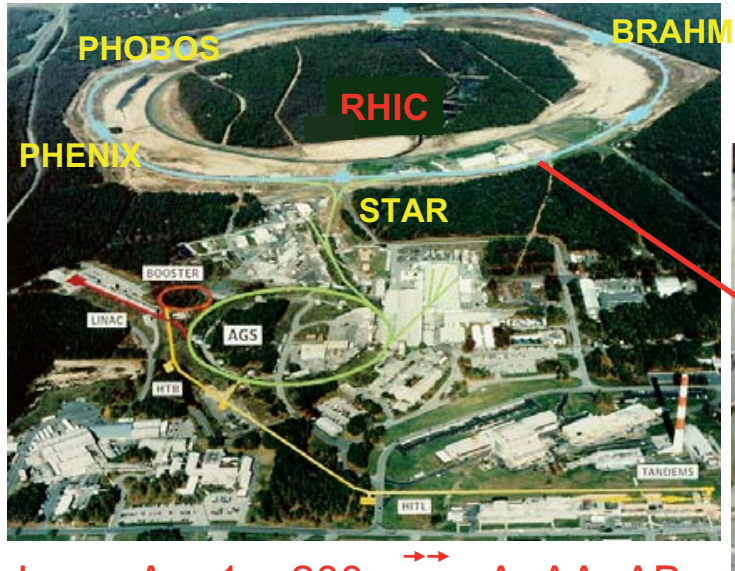

lons: $\mathrm{A}=1 \sim 200, \overrightarrow{\mathrm{pp}}, \mathrm{pA}, \mathrm{AA}, \mathrm{AB}$

Two Concentric Superconducting Rings

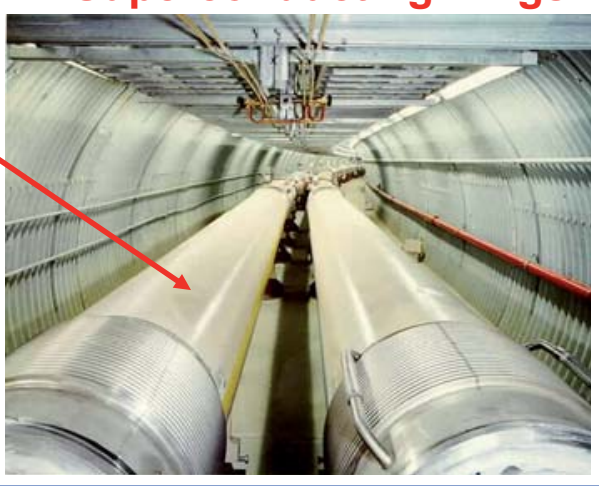

\begin{tabular}{|c|c|c|}
\hline Design Performance & $\underline{A u}+\mathrm{Au}$ (Now) & (Now@200) \\
\hline $\operatorname{Max} \sqrt{ } s_{n n}$ & $200 \mathrm{GeV}$ & $500 \mathrm{GeV}$ \\
\hline$L\left[\mathrm{~cm}^{-2} \mathbf{s}^{-1}\right]$ & $2 \times 10^{26} \quad\left(3.6 \times 10^{27}\right)$ & $1.4 \times 10^{31} \quad\left(3.5 \times 10^{31}\right)$ \\
\hline Interaction rates & $1.4 \mathrm{khz} \quad(\sim 36 \mathrm{khz})$ & $300 \mathrm{khz} \quad(\sim 750 \mathrm{khz})$ \\
\hline
\end{tabular}




\section{The (evolving) STAR detector}

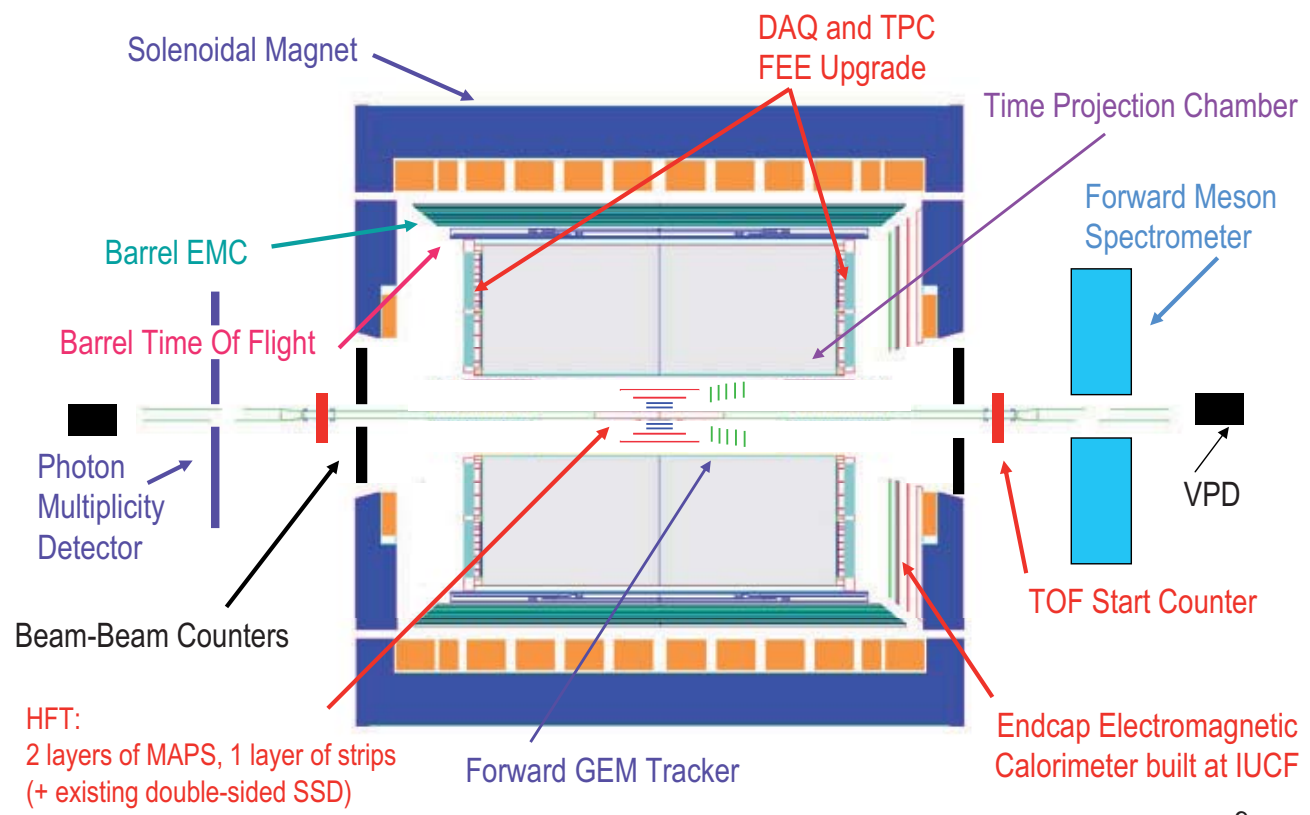

TJH: JINR Heavy lon Summer School

July 14-26 Dubna, Russia

\section{From $p+p$ to $A u+A u$ in the STAR TPC}

$p+p \rightarrow$ jet+jet

(STAR@RHIC)

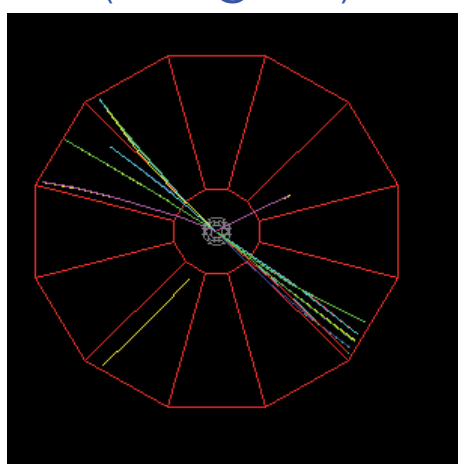

$\mathrm{Au}+\mathrm{Au} \rightarrow \mathrm{X}$

(STAR@RHIC)

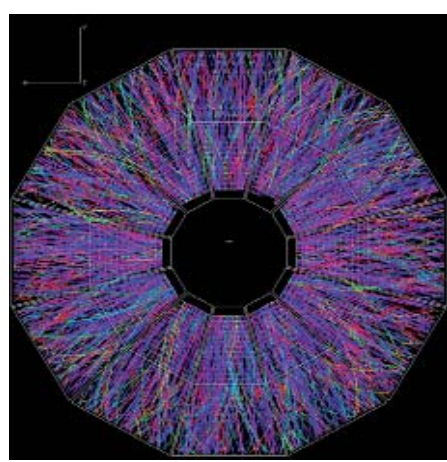




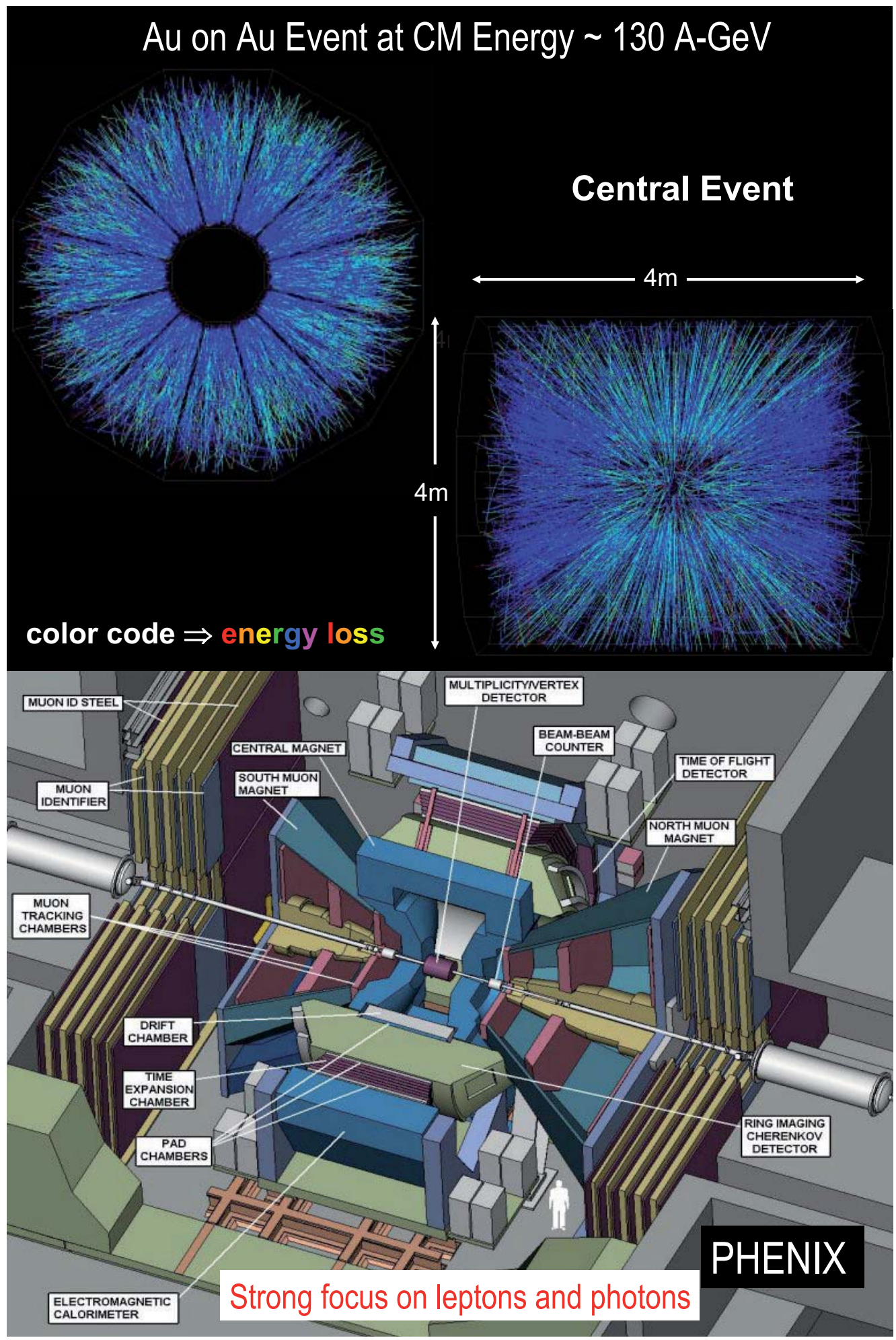




\section{Time line of a relativistic heavy ion collision}

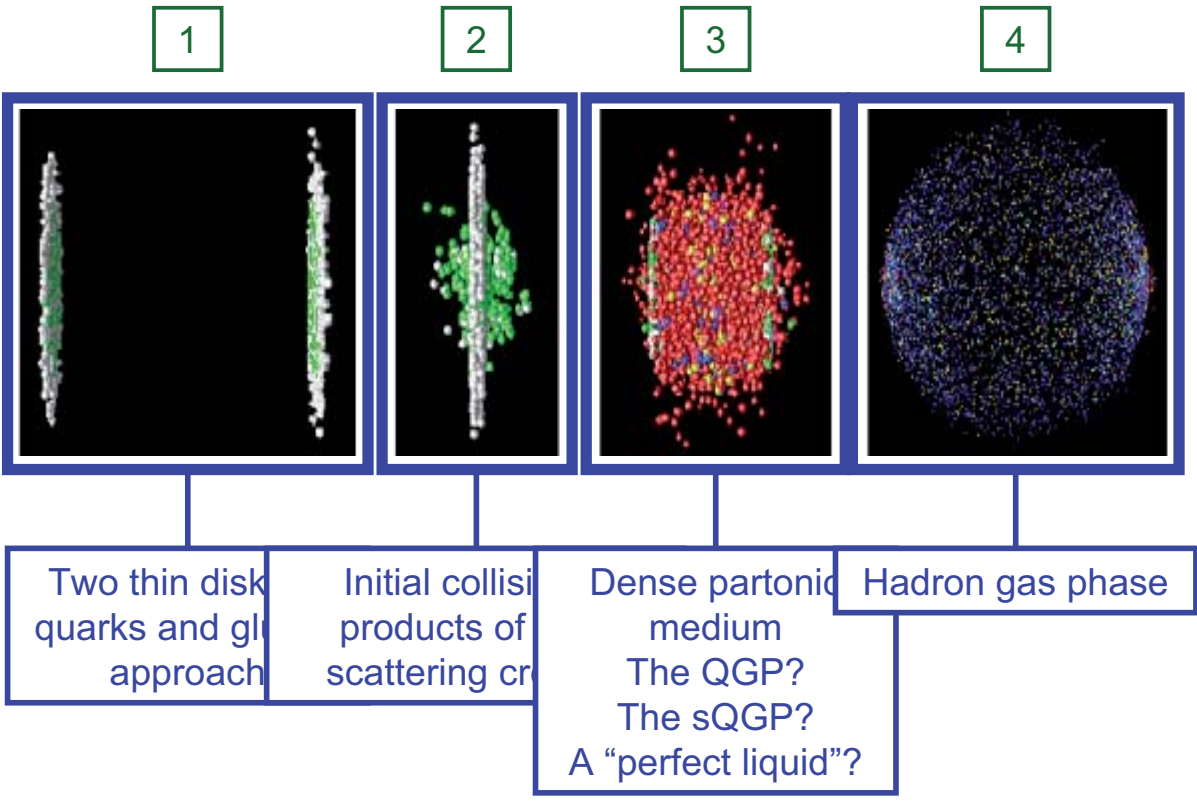

\section{Kinematics for colliders}

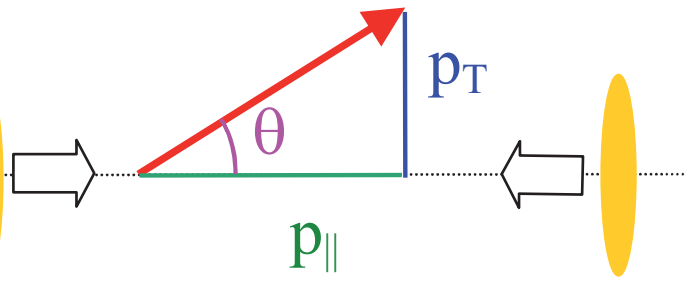

Pseudo-rapidity: $\quad \eta=-\ln [\tan (\theta / 2)] \approx y$

Transverse momentum $\left(p_{T}\right)$ and pseudorapidity $(\eta)$ provide a convenient description

$$
\begin{gathered}
p_{z}=p_{T} \sinh (\eta) \\
p_{t o t}=p_{T} \cosh (\eta)
\end{gathered}
$$

Mid-rapidity: $\quad \eta=0$, perpendicular to the incident beams

$\eta=4: \quad$ Scattering at $\theta=2.1^{\circ}$ in the CM (or lab) frame 


\section{A few more terms in heavy-ion physics}

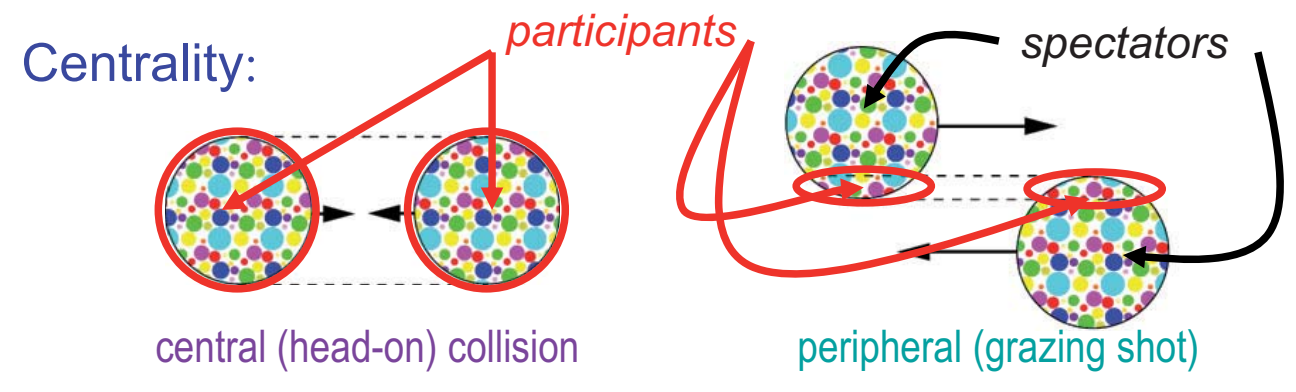

Number of Participants ( $\left.\mathrm{N}_{\text {part }}\right)$ :

- Number of nucleons participating in the collision

- $p+p$ collisions $=2$

- central Au+Au $350 \quad(A u=79 p+118 n=197)$

- Used to describe geometry of a collision

What scale of excitation is required to liberate quarks and gluons?

- In a normal nucleus the nucleon density is $\sim .16 \mathrm{fm}^{-3} \rightarrow \sim .15 \mathrm{GeV} \mathrm{fm}^{-3}$

- We might expect things to "get interesting" when two nucleons are more than $50 \%$ overlapping

- In this case the energy density is $\varepsilon \sim 2 \mathrm{GeV} / \underline{4} \pi \mathrm{r}^{3} \sim 1 \mathrm{GeV} / \mathrm{fm}^{3}$ where $r_{\text {nucleon }} \sim 0.8 \mathrm{fm}$

- More refined estimates indicated when $\varepsilon \sim 2-3 \mathrm{GeV} / \mathrm{fm}^{3}$ we should see a transition

How does that compare with the heating in a nucleus-nucleus collision? 
One way to tell is to estimate of the energy density at RHIC EMCAL

by seeing how much energy is "redirected" sideways (how much transverse energy $\left(E_{T}\right)$ there is)
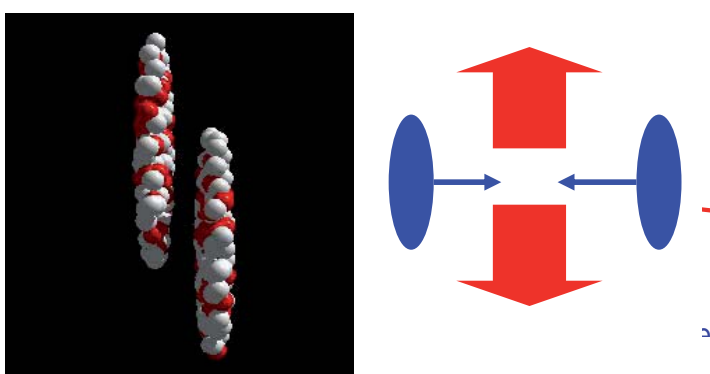

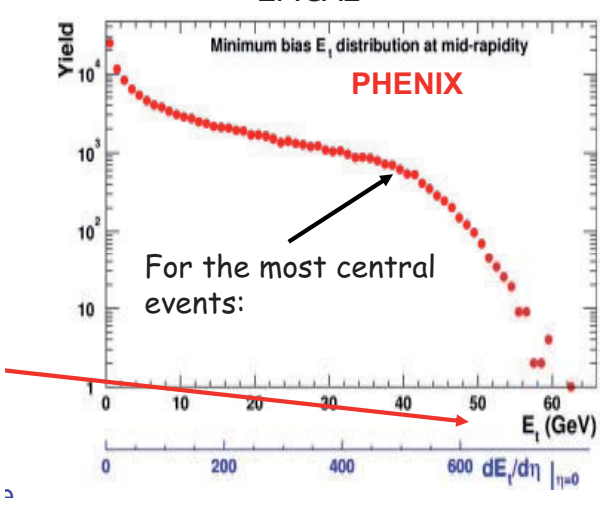

We are definitely in the "ball park" where we expect a transition

\section{What discoveries has the first phase at RHIC yielded?}




\section{How can we quantify the elliptic flow}

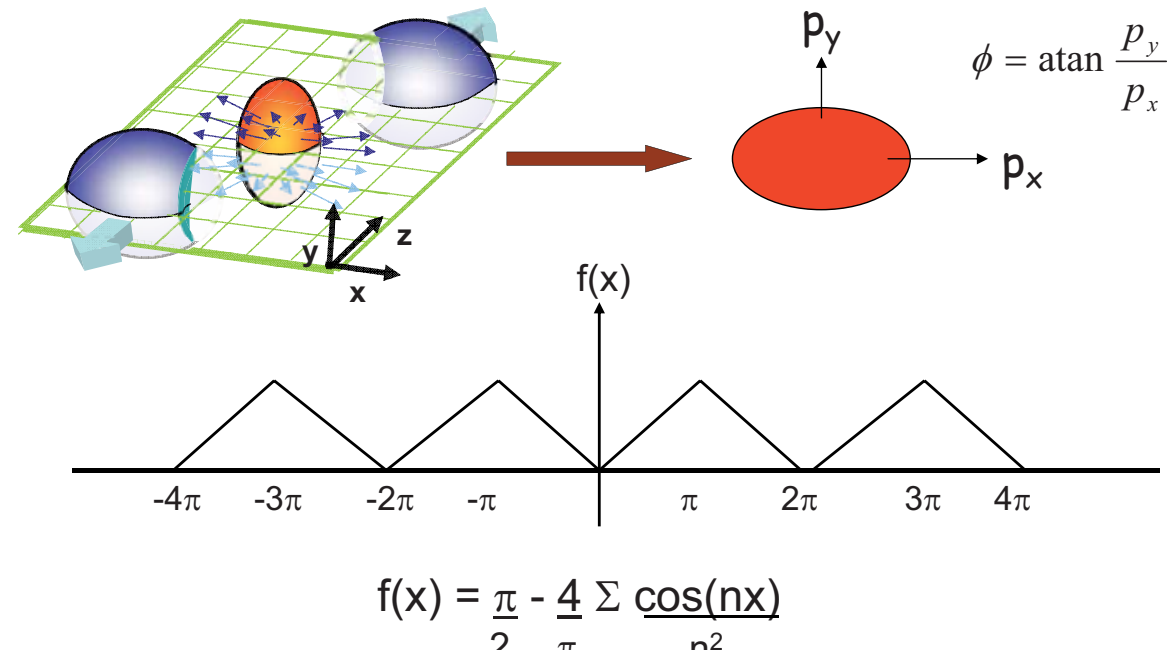

Elliptic flow establishes there is strongly interacting matter at $t \sim 0$

\section{Gas of weakly/strongly interacting Li atoms}

M. Gehm et al, Science 298, 2179

- excite Feshbach resonance
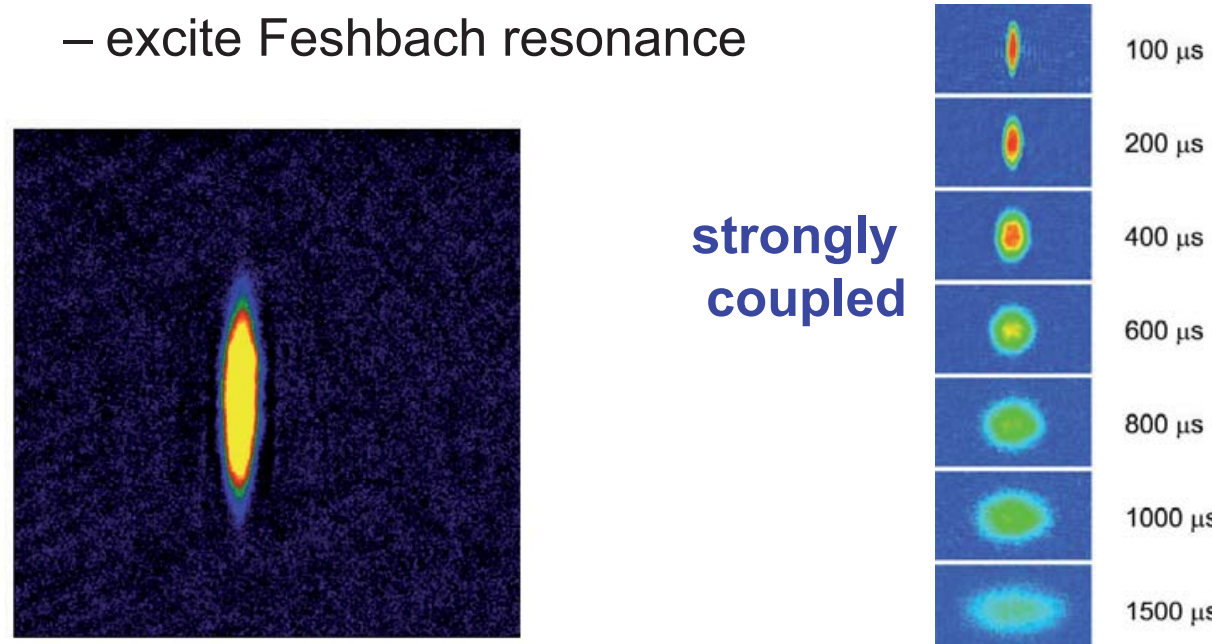

weakly coupled

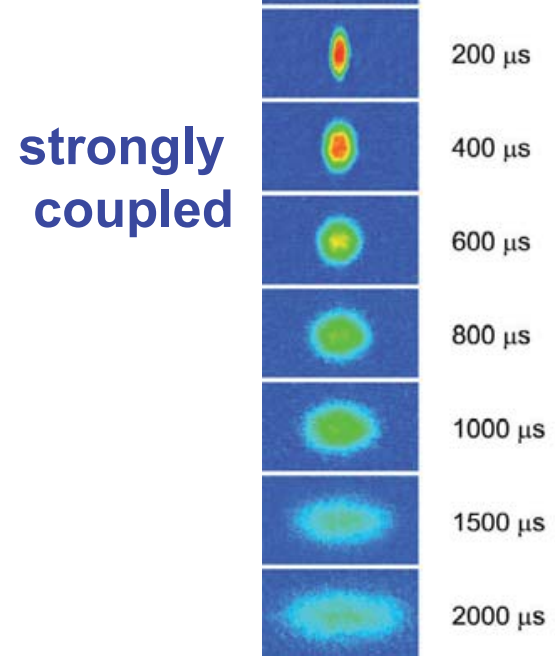




\section{Is there elliptic flow at RHIC?}

\section{Yes! First time hydrodynamics without any viscosity describes heavy ion reactions.}

- Hydrodynamic calculations as $\leq_{\omega} 0.3$

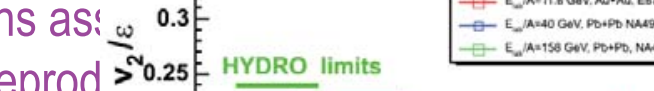
$\mathrm{p}_{\mathrm{T}} \sim 1.5 \mathrm{GeV} / \mathrm{c}$

- Same calculations fit the radia

- Elliptic flow saturates the hydre

- Very rapid thermalization, very

- A perfect fluid?

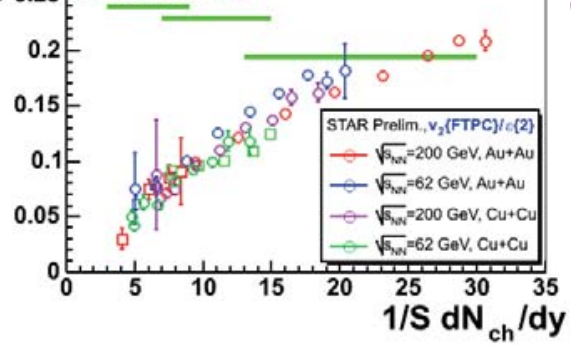

Thermalization time $\underline{\tau<1 \mathrm{fm} / \mathrm{c} \text { and } \underline{\varepsilon}=20 \mathrm{GeV} / \mathrm{fm}^{3}}$

What is the viscosity?

How perfect is our liquid?

A. Nakamura and S. Sakai, hep-lat/0406009

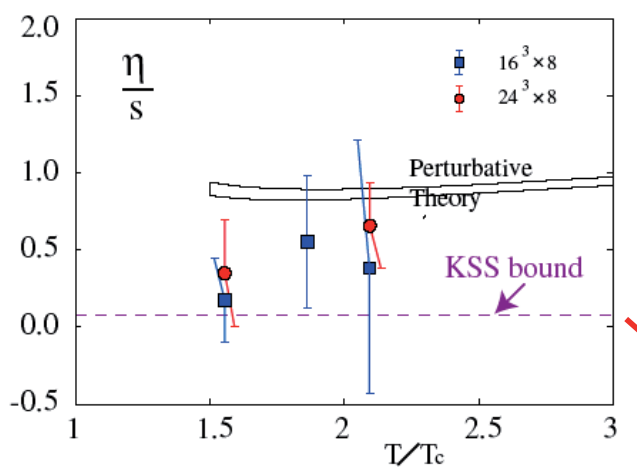

Kovtun, Son, Starinets, Phys. Rev. Lett 94, 2005

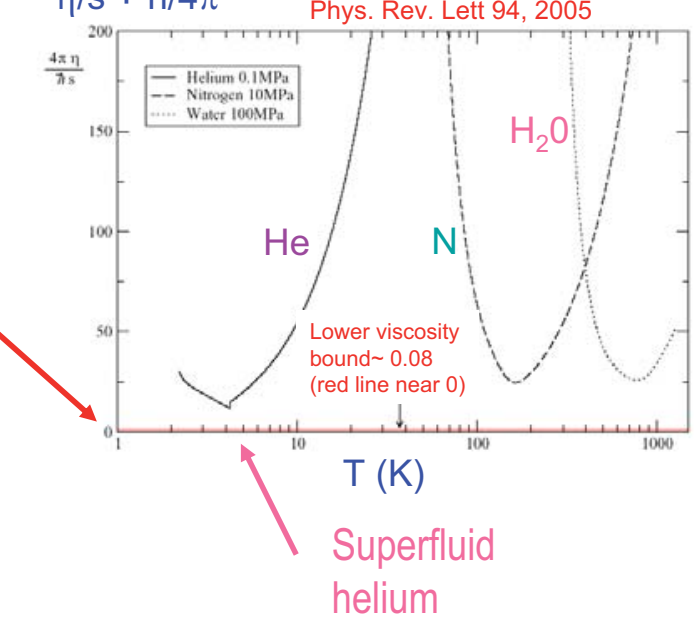

22 


\section{How to Quantify $\eta / s$ at RHIC?}

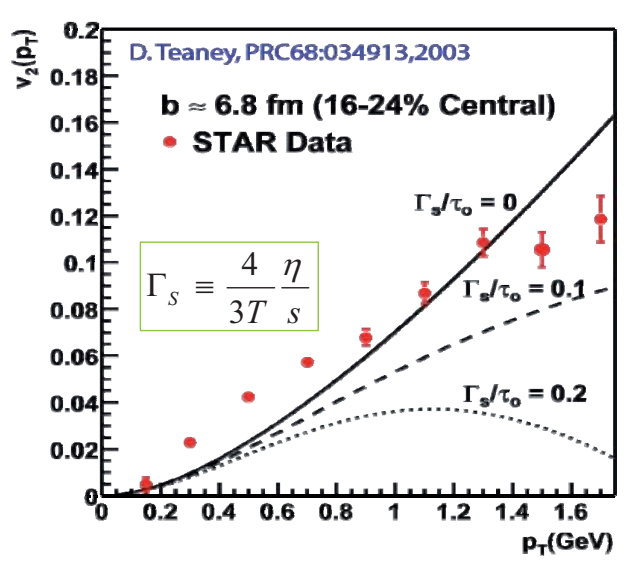

Ultimately Needed:

- Continued progress on viscous relativistic hydrodynamic theory

- Radial, directed, elliptic flow measurements for several identified hadron species. Particularly valuable:

- Multi-strange hadrons $\varphi, \equiv, \Omega$ (reduced coupling to hadron gas phase) to determine viscous effects in the hadronic phase

- D mesons (establish thermalization time scale)

B meson D meson

$\Gamma_{\mathrm{S}}=$ sound attenuation length

( mean free path)

For reasonable $\mathrm{T}\left(\sim 2 \mathrm{~T}_{\mathrm{C}}\right)$ and $\tau$

$(\sim 1 \mathrm{fm} / \mathrm{c})$ data suggest $\eta / \mathrm{s}<0.3$

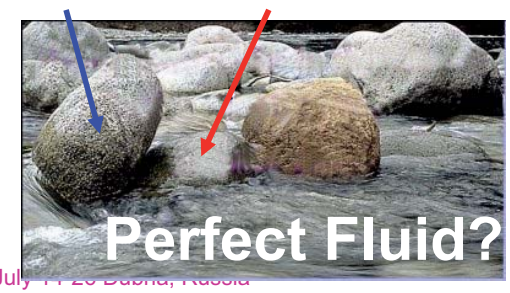

\section{String Theory?}

What could this have to do with our physics?

The Maldacena duality, know also as AdS/CFT correspondence, has opened a way to study the strong coupling limit using classical gravity where it is difficult even with lattice Quantum Chromodynamics.

It has been postulated that there is a universal lower viscosity bound for all strongly coupled systems, as determined in this dual gravitational system.

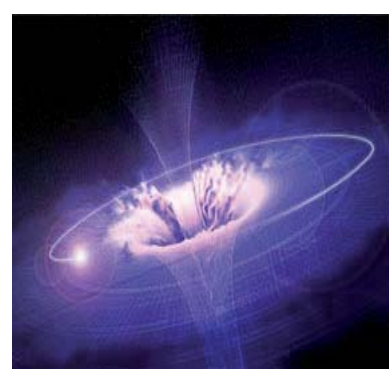

July 14-26 Dubna, Russia 
What other dynamical effects allow access the properties of this fleeting matter?

How do we look inside something we can't physically take apart?

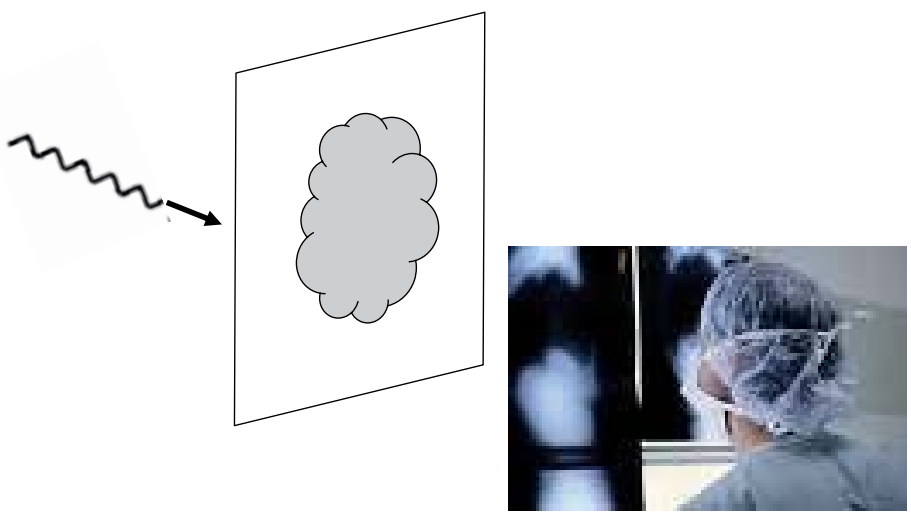

We use a penetrating probe (one that can go through) which is well calibrated

In the case of RHIC, the probe is the quarks and gluons in the beam to start with- inside the protons and neutrons of the Au nucleus !

TJH: JINR Heavy lon Summer School July 14-26 Dubna, Russia

They allow us to search for new physics. The calibrated probe is the back to back correlation which results when the quarks \& gluons in the two beams scatter

What do we expect?

Looking along the beam line

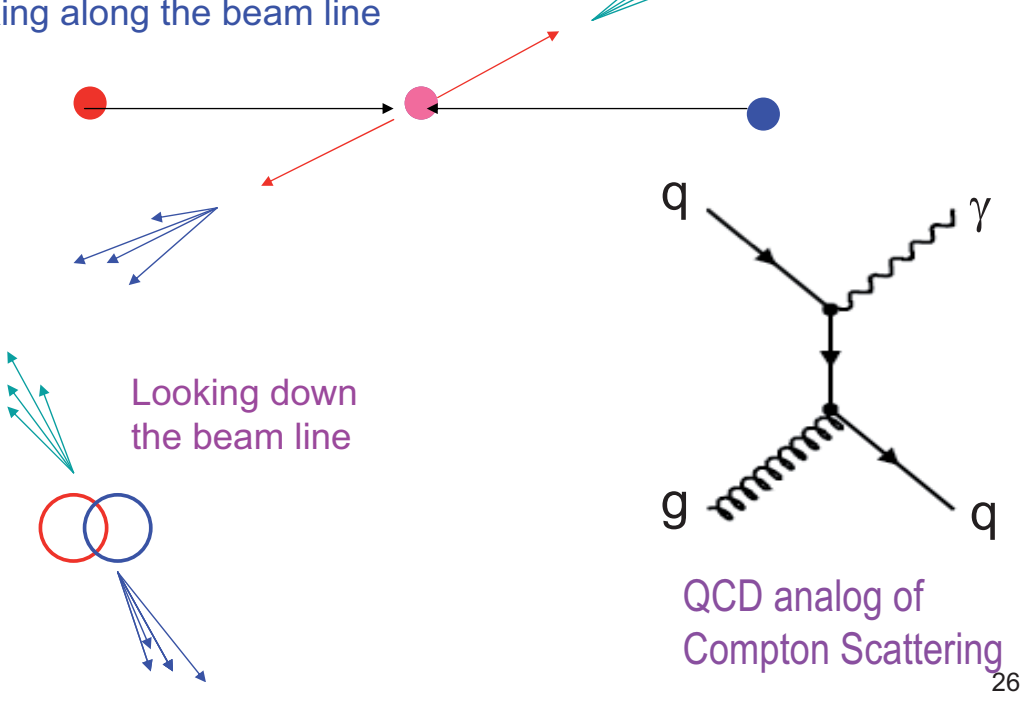




\section{From $p+p$ to $A u+A u$ in the STAR TPC}
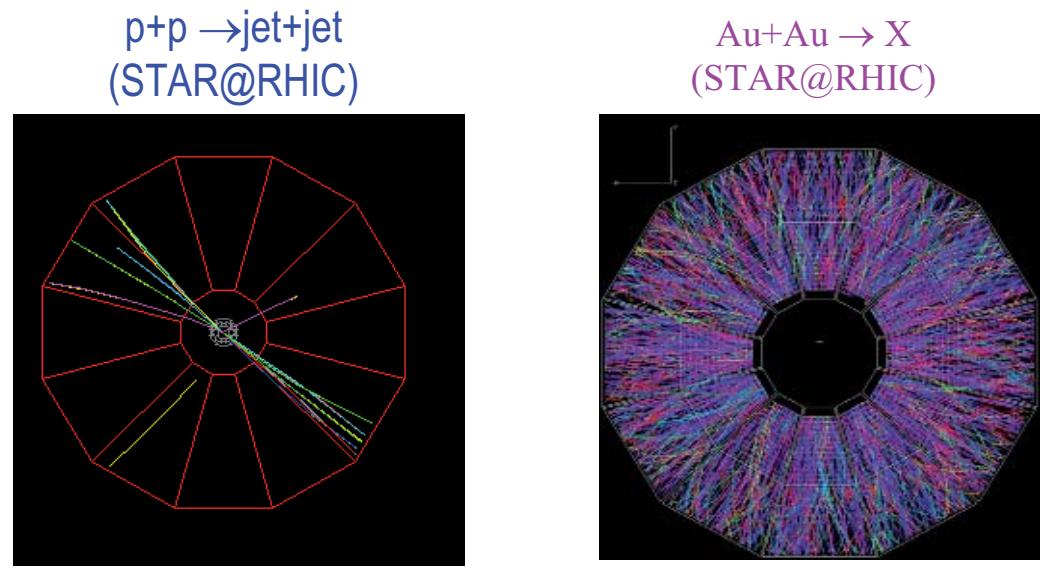

In principle, there is a lot of information needed to understand High $\mathrm{p}_{T}$ Particle Production in A+A Collisions $\frac{d N_{A B}^{h}}{d y d^{2} p_{T}}=K \sum_{a b c d} \int d x_{a} d x_{b} \int d^{2} \mathbf{k}_{a} d^{2} \mathbf{k}_{b}$ (According to $\mathrm{pQCD}$...)

$\times f_{a / A}\left(x_{a}, Q^{2}\right) f_{b / B}\left(x_{b}, Q^{2}\right) \quad$ Parton Distribution Functions $\times g\left(\mathbf{k}_{a}\right) g\left(\mathbf{k}_{b}\right) \quad$ Intrinsic $\mathbf{k}_{\mathrm{T}}$, Cronin Effect $\times S_{A}\left(x_{a}, Q_{a}^{2}\right) S_{B}\left(x_{b}, Q_{b}^{2}\right) \quad$ Shadowing, EMC Effect $\times \frac{d \sigma}{d \hat{t}}(a b \rightarrow c d)$ $\times \int_{0}^{1} d \varepsilon P(\varepsilon) \frac{z_{c}^{*}}{z_{c}}$ Hard-scattering cross-section $\frac{D_{h / c}^{0}\left(z_{c}^{*}, Q_{c}^{2}\right)}{z_{c}}$ $\pi z_{c}$

Partonic Energy Loss 


\section{But using our calibrated probe we can try something simpler to start}

Looking along the beam line
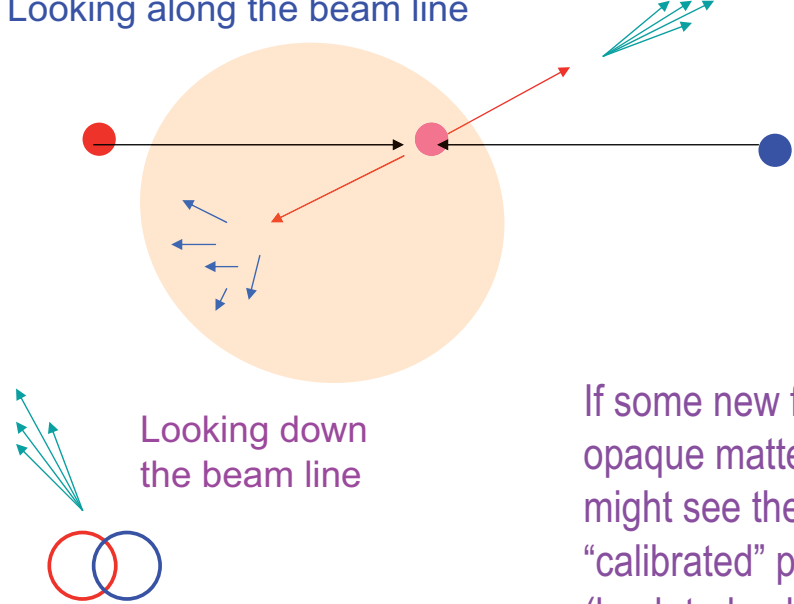

If some new form of dense,

Looking down

the beam line

opaque matter is formed, we might see the effects on our "calibrated" probe (back-to-back correlation)

\section{A second discovery: jet quenching}

An "inclusive" measure:

the "Nuclear Modification Factor:"

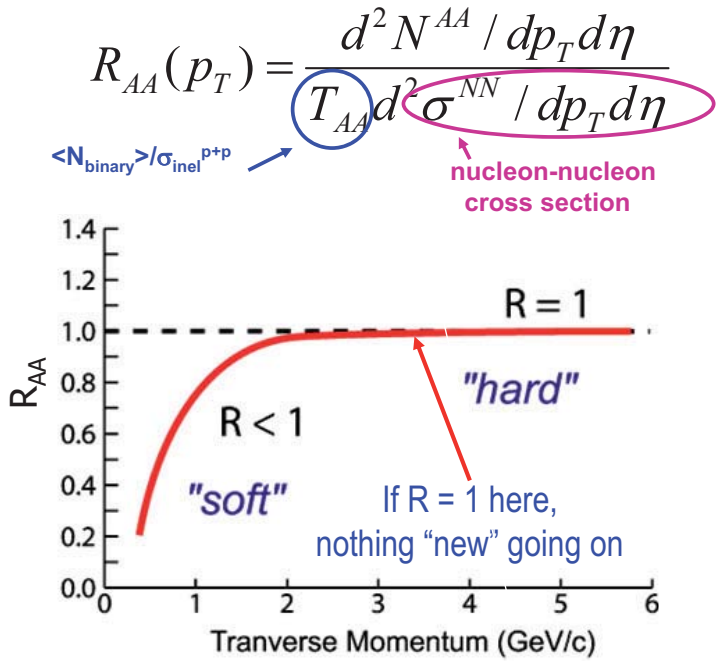

TJH: JINR Heavy lon Summer School
A second test: back-to-back correlations from di-jets jets

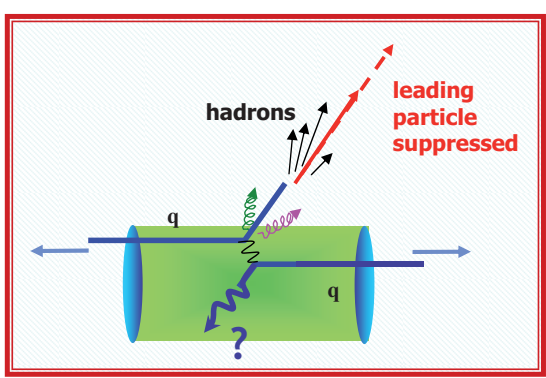

Will our calibrated penetrating probe go through the same way? -- or will it be quenched ? -- 


\section{Hard scattering at RHIC and NLO PQCD}

PRL 91, 241803

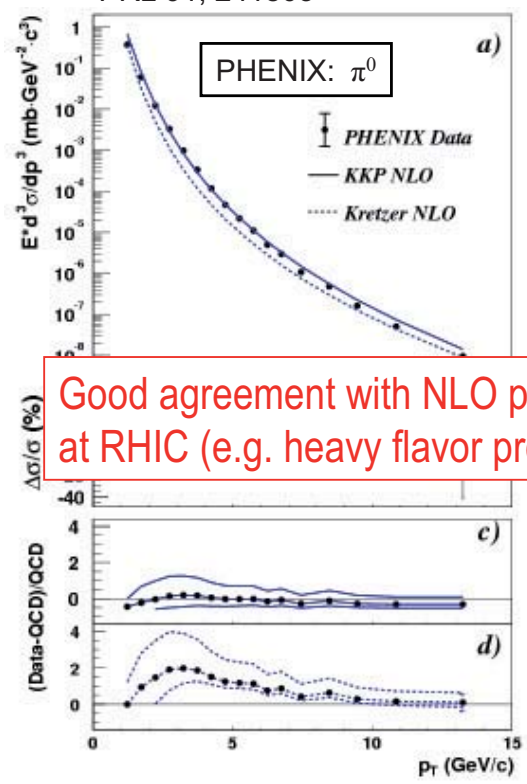

hep-ex/0608030

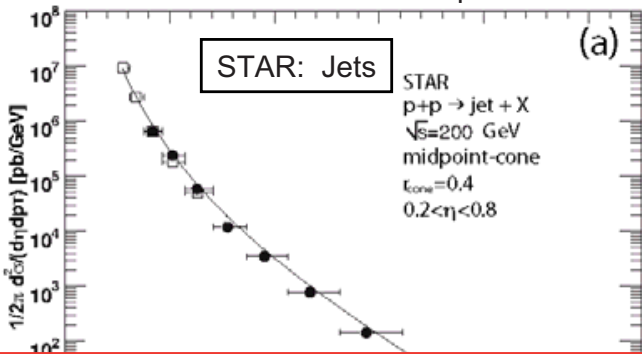

(a)

\section{.}

$1 n^{2}$ E

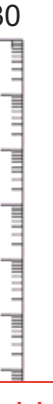
roduction...)

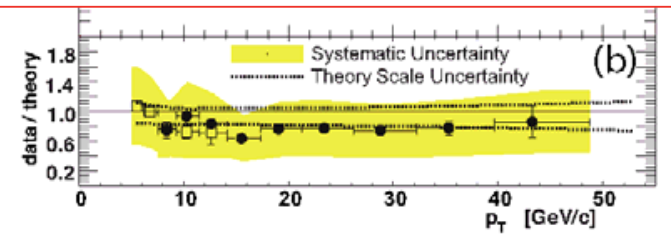

In central Au+Au collisions something dramatically new occurs: jet quenching $R_{A A}\left(p_{T}\right)=\frac{d^{2} N^{A A} / d p_{T} d \eta}{T_{A A} d^{2} \sigma^{N N} / d p_{T} d \eta}$ Nuclear Modification Factor

Binary collision scaling

Particles which are made up from colored quarks and gluons are strongly suppressed

Direct photons are not

The contsant level of suppression indicates the effect occurs before the final particles are made (hadronization) Au+Au (central collisions): $\sqrt{s_{\mathrm{NN}}}=200 \mathrm{GeV}$

- Direct $\gamma$ (PHENIX Preliminary)

$* \quad$ Inclusive $\mathrm{h}^{ \pm}$(STAR)

- $\pi^{0}$ (PHENIX Preliminary)

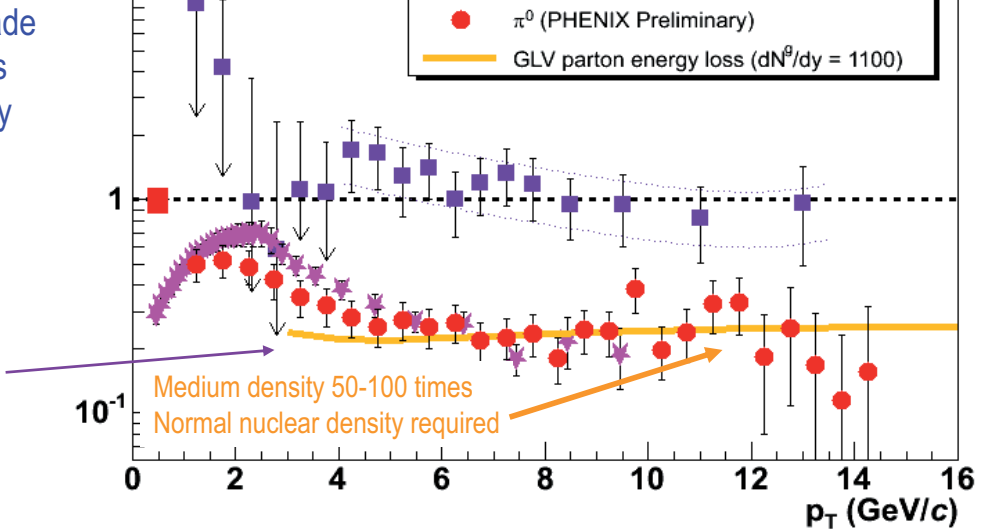




\section{Jet quenching at RHIC}

The effect is seen even more dramatically in dihadron correlations: recoiling jets are strongly modified due to quenching

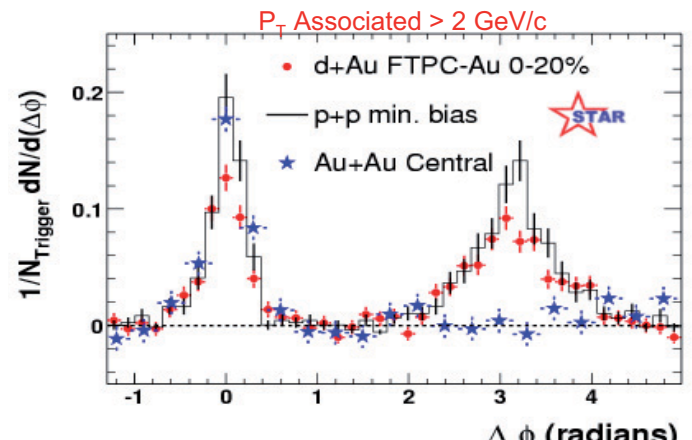

Unequivocally, a new phenomena has been observed

- In central Au+Au collisions:

Not everything is understood:

Electrons from the semi-leptonic decay of charm + bottom appear as suppressed as particles containing light quarks. That raises new questions (one subject of tomorrows seminar)

But evidence for jet quenching in some new form of opaque matter is unequivocal

Additional evidence from something that was not predicted

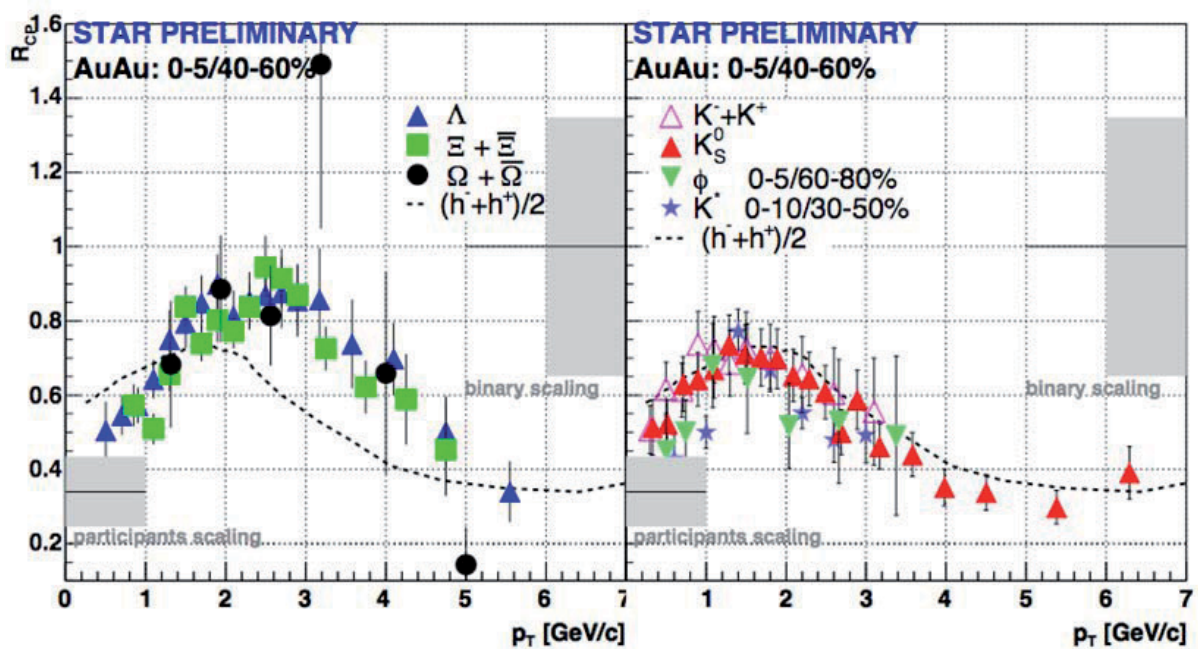

The mesons (quark-antiquark) and the baryons (3 quarks) exhibit two distinct behaviors below transverse momenta of $\sim 6 \mathrm{GeV} / \mathrm{c}$ $\left(\mathrm{K}^{*}, \phi\right.$ which are as heavy as the proton follow the mesons!) 
Same "splitting" of mesons/baryons if you look at elliptic flow
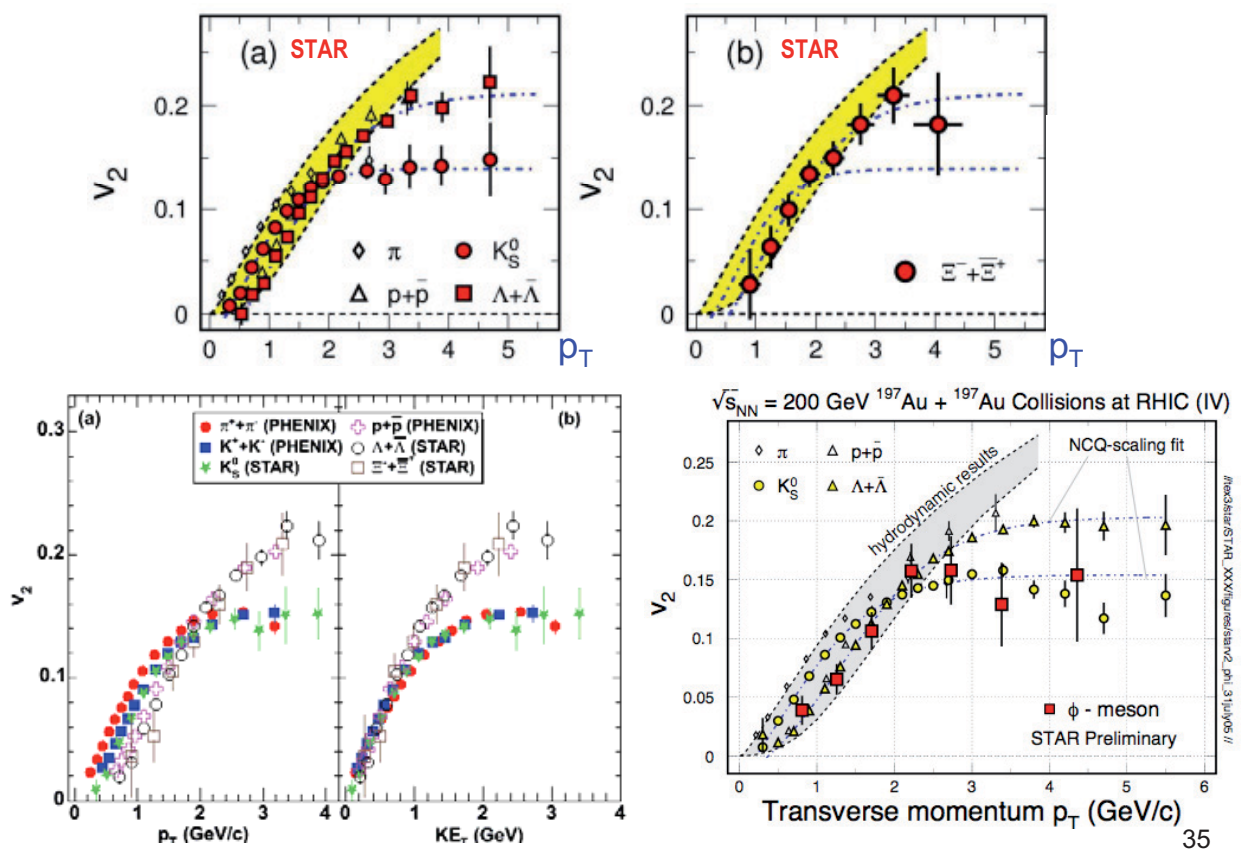

TJH: JINR Heavy Ion Summer School

July 14-26 Dubna, Russia

\section{What if quarks coalesce to make hadrons?}

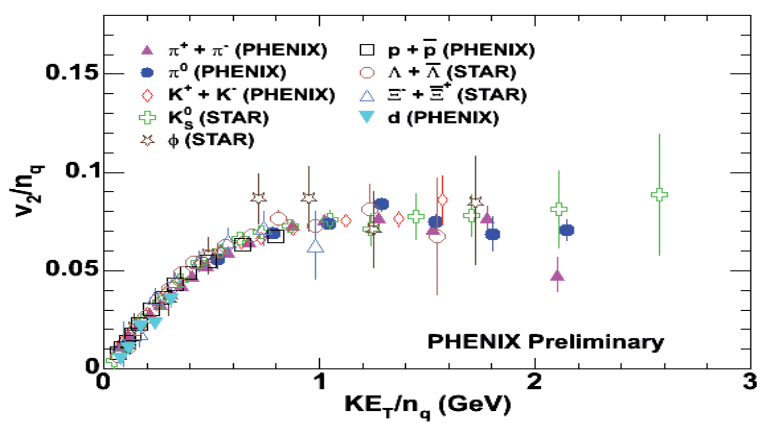

- $v_{2}$ obeys constituent quark scaling

- Hadronization through coalescence

- Evidence for flowing quarks

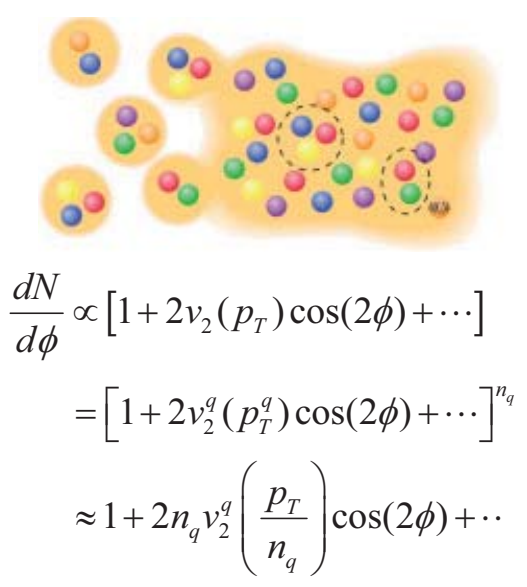




\section{A remarkable scaling of the "fine structure" of elliptic flow is observed}
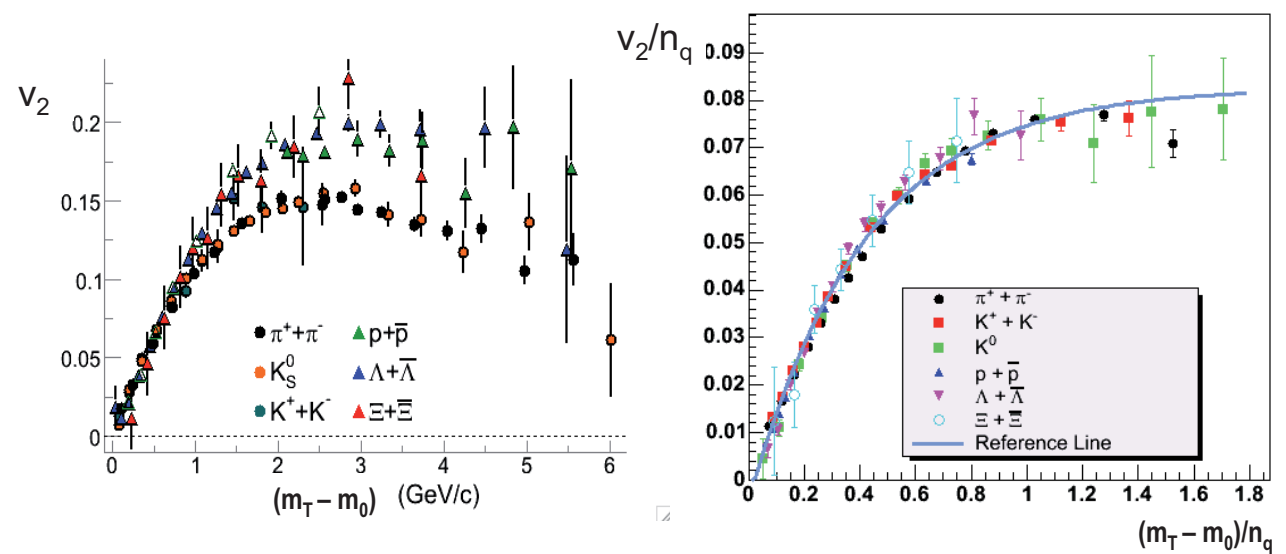

Fluid $\rightarrow$ QuasiParticles $\rightarrow$ Hadrons

Evidence for fluid breaking up into quasiparticles with quantum numbers of quarks before hadrons

\section{Supporting Evidence :}

For a thermalized system of quarks (describable by thermodynamic properties such as temperature and chemical potential) then the ratios of the yields of particles distilled (hadronized) out of this quark soup should be predictable by statistical thermodynamics. Is it?
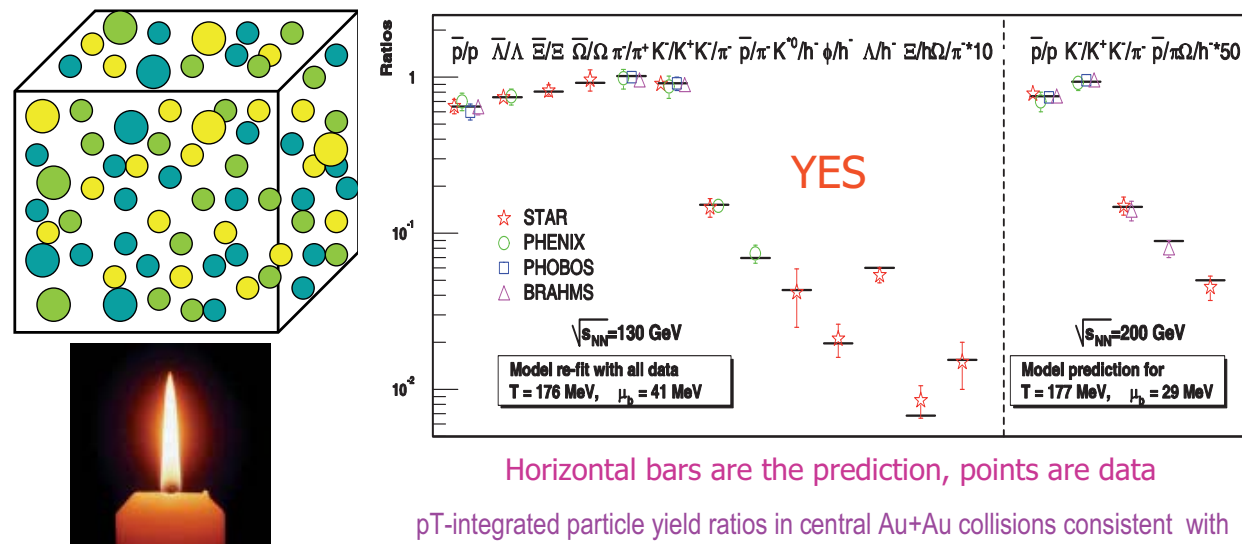

Horizontal bars are the prediction, points are data

pT-integrated particle yield ratios in central Au+Au collisions consistent with Grand Canonical Stat. distribution @ Tch $=(160 \pm 10) \mathrm{MeV}, \mu \mathrm{B} \approx 25 \mathrm{MeV}$, across $u, d$ and s quark sectors. Inferred Temp. consistent with Tcrit (LQCD) $\Rightarrow$ phase transition 
Three major discoveries at RHIC which point unequivocally to a new state of strongly interacting quark-gluon matter

The hottest, densest matter yet examined in the laboratory

It is highly opaque to colored probes- quarks and gluons but not to photons

It flows as a relativistic quantum liquid with minimal shear viscosity

It produces copious mesons and baryons with yield ratios and flow properties that suggest their forme via coalescence of valence quarks from a hot thermal bath.

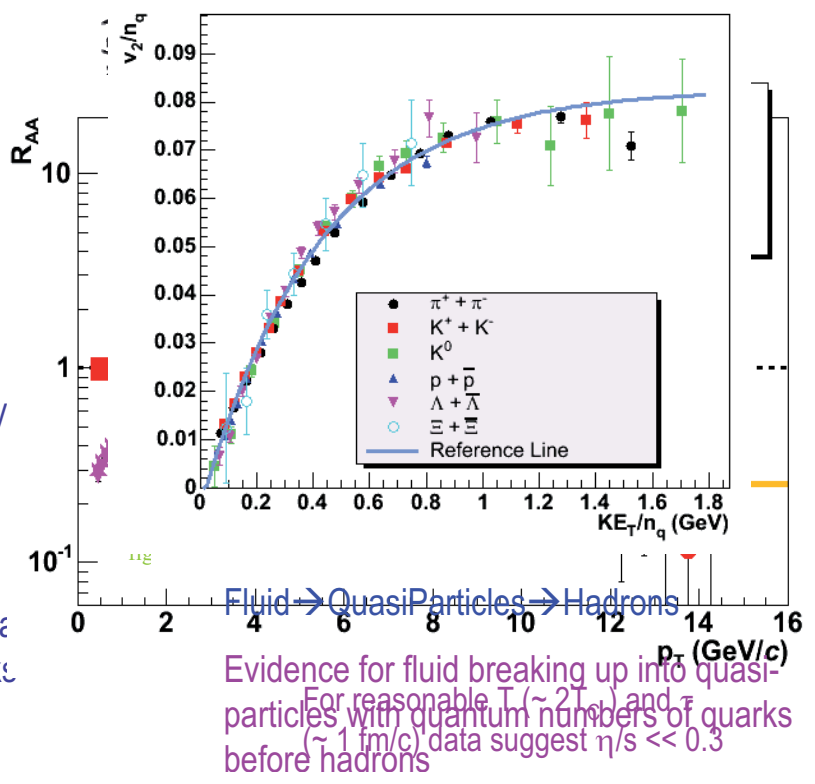

These phenomena were not observed at the SPS (some were not even predicted) and they constitute important new discoveries

\section{Are we done?}

\section{No, only just begun}

New questions have emerged from exploring this terra incognita 


\section{Progress on the basics: the charm cross-section}

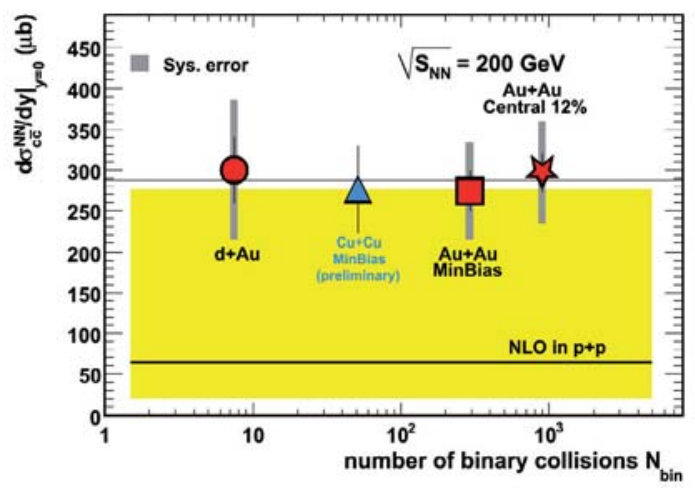

- $\quad$ Charm cross section scales with the number of binary collisions

- Multiple measurements in different channels all give the same result

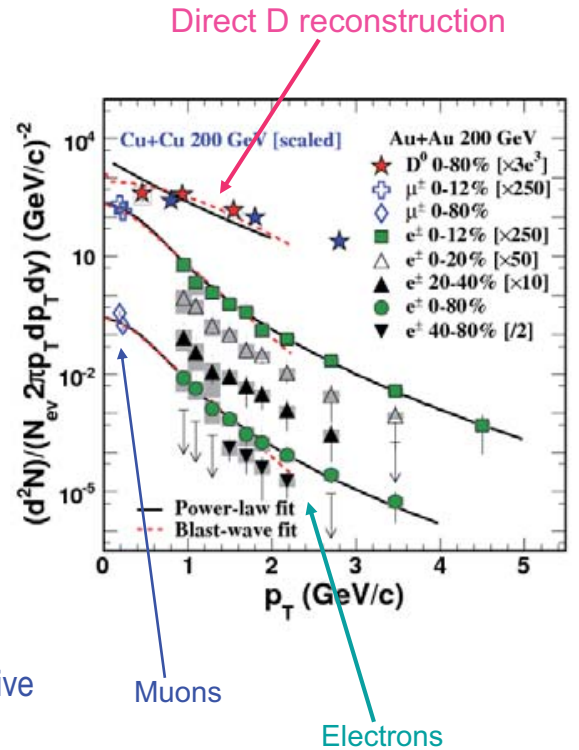

41

TJH: JINR Heavy Ion Summer School

July 14-26 Dubna, Russia

A new puzzle which emerges:

Initially there was a reasonably strong consensus that the suppression was basically understood: radiative energy loss in a medium 50-100 times normal nuclear matter density

Then these measurements were extended to the heavy quark sector (c, b)

by studying suppression of electrons from their semi-leptonic decays

\section{Heavy quark energy loss}

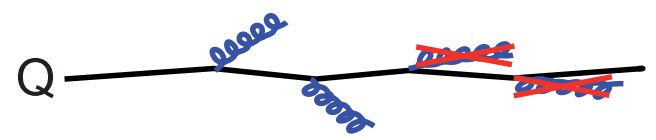

Dokshitzer, Khoze, Troyan, JPG 17 (1991) 1602.

Dokshitzer and Kharzeev, PLB 519 (2001) 199.

- In vacuum, gluon radiation suppressed at $\theta<m_{\mathrm{Q}} / E_{\mathrm{Q}}$

- "dead cone" effect: heavy quarks fragment hard into heavy mesons

Dead cone also implies lower heavy quark energy loss in matter: (Dokshitzer-Kharzeev, 2001)

$$
\left.\omega \frac{\mathrm{d} I}{\mathrm{~d} \omega}\right|_{\text {HEAVY }}=\frac{\left.\omega \frac{\mathrm{d} I}{\mathrm{~d} \omega}\right|_{\text {LIGHT }}}{\left(1+\left(\frac{m_{Q}}{E_{Q}}\right) \frac{1}{\theta^{2}}\right)^{2}}
$$




\section{Heavy flavor suppression via b,c $\rightarrow$ e+X}
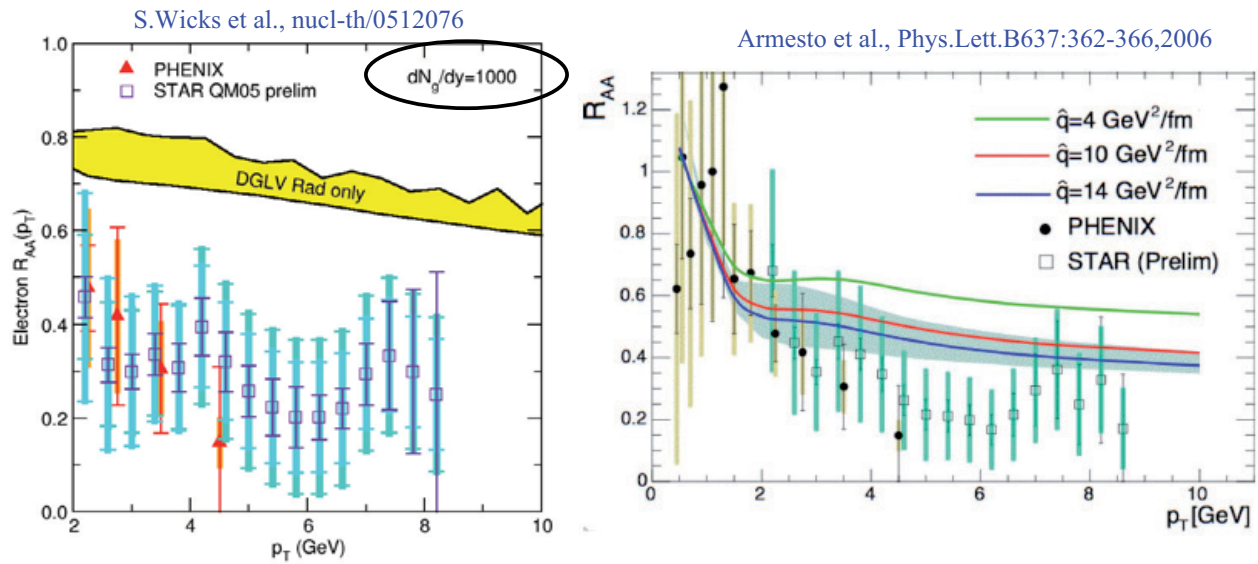

$\mathrm{R}_{\mathrm{AA}}$ (non-photonic electrons) $\sim 0.2 \sim \mathrm{R}_{\mathrm{AA}}\left(\pi^{0}\right) ! !$

Gluon density/qhat constrained by light quark supression+entropy density (multiplicity)

$\Rightarrow$ under-predicts electron suppression

$\Rightarrow$ charm vs beauty? elastic energy loss? ...?

\section{Surprising results on suppression of High- $\mathrm{p}_{\mathrm{T}}$ Charm via Electrons}

Ratio of charm spectra in Au+Au to $p+p$ normalized by No. of binary collisions \& comparison with models of PQCD energy loss primarily based on radiation of gluons

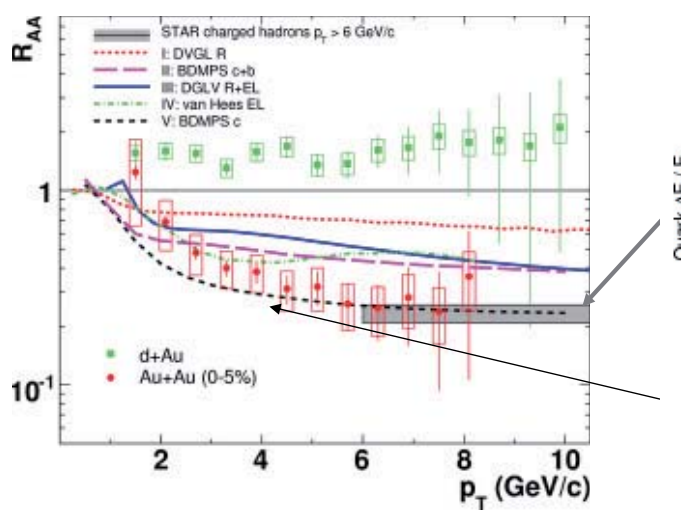

Results caused a shift of paradigm on importance of collisional energy loss S.Wicks et al., nucl-th/0512076

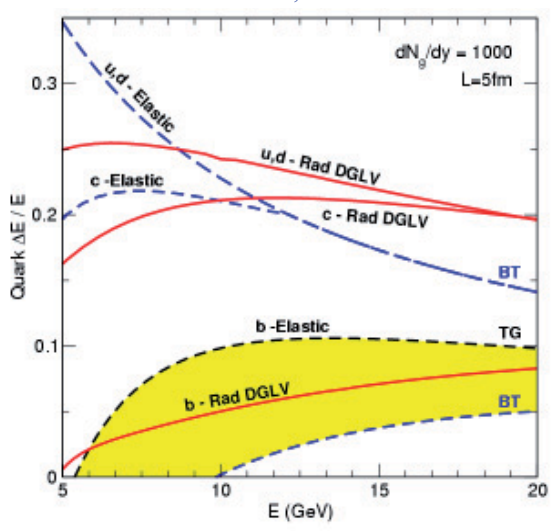

- Measurement of non-photonic electrons from semileptonic $D$ decays show substantial suppression in central Au+Au collisions comparable to that from light mesons

- Describing the suppression is difficult for models $\rightarrow$ theory paradigm shift on radiative energy loss, collisional E-loss, fragmentation and dissociation in medium?

- Energy loss models need to be revisited! 
Additional insight is possible using electron tagged correlations
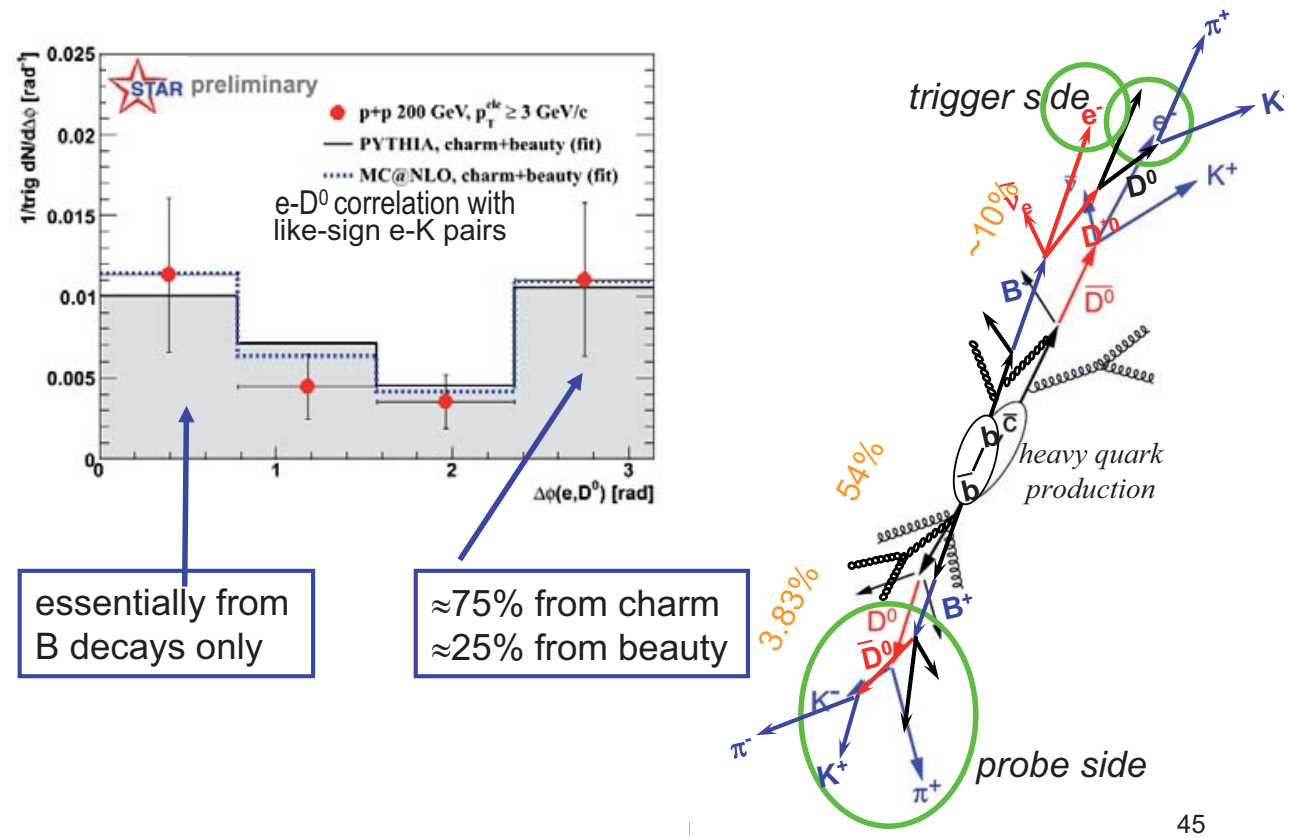

July 14-26 Dubna, Russia

\section{Additional insight from heavy flavor correlations}

All measurements in $p+p$ at sqrt(s) $=200 \mathrm{GeV}$

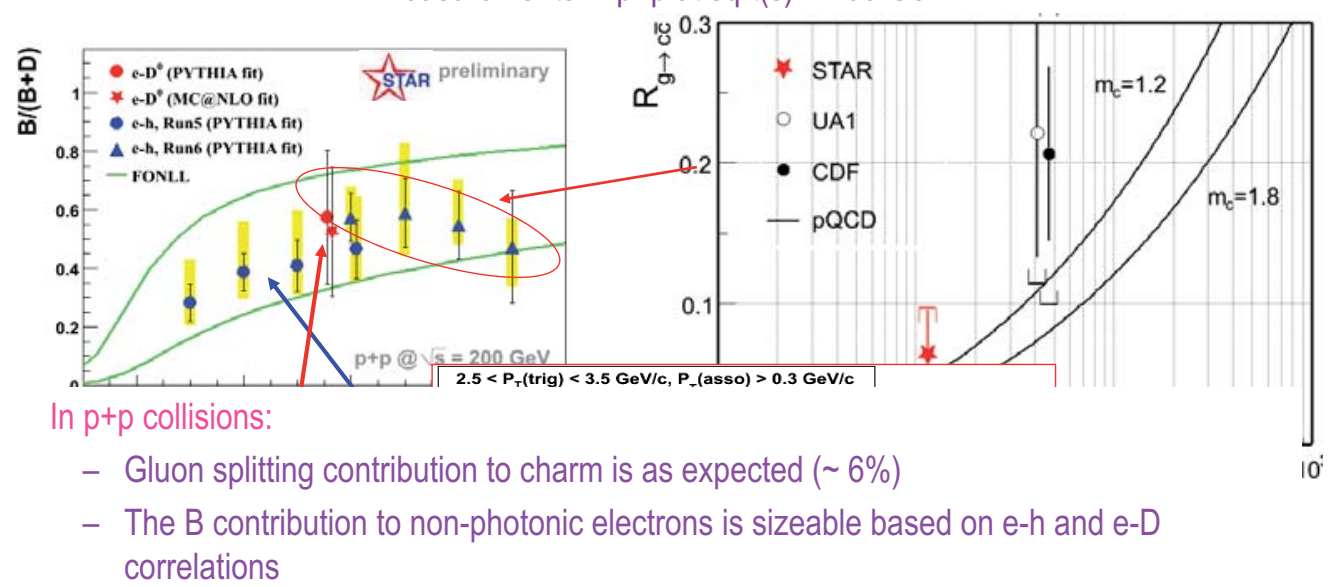

Taken together with suppression of non-photonic electrons in $A u+A u$, this suggests significant suppression of non-photonic electrons from bottom in the medium 


\section{Insight from heavy flavor correlations in $p+p$}

All measurements in $p+p$ at sqrt(s) $=200 \mathrm{GeV}$

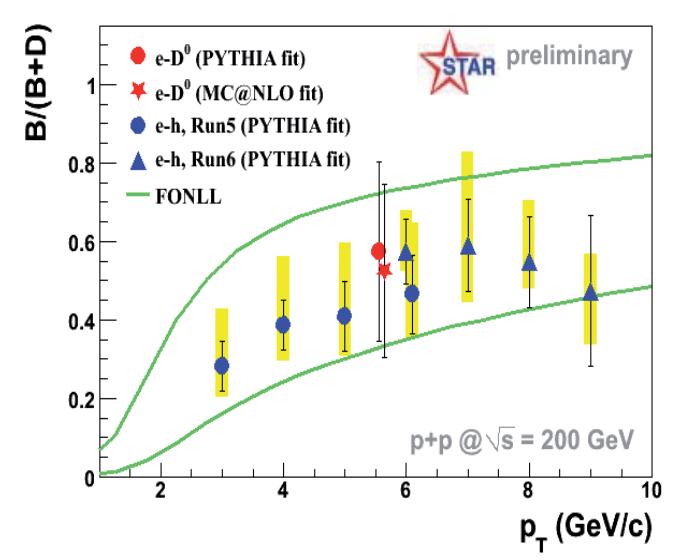

In $p+p$ collisions:

- The B contribution to nonphotonic electrons is sizeable based on e-hadron and e-D meson correlations

Taken together with suppression of non-photonic electrons in $A u+A u$, this suggests significant suppression of non-photonic electrons from bottom in the medium

This may be hinting our paradigm needs to change

\section{Possible example of paradigm shift at RHIC}

- From Dmitri Kharzeev on PQCD energy loss: "if it is really true that bottom is suppressed, there's just no way.."

\section{First glimpses of a new paradigm?}

BNL-NT-07/47

RBRC-703

Universal properties of bulk viscosity near the QCD phase transition

Frithjof Karsch $^{a}$, Dmitri Kharzeev ${ }^{a}$ and Kirill Tuchin ${ }^{b, c}$

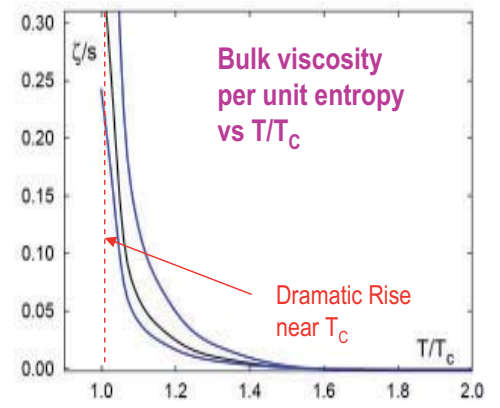

arXiv:0711.0914v1 [hep-ph] 6 Nov 2007
Bulk viscosity of hot qgp in the presence of light quarks from lattice data on QCD equation of state

- Large Bulk Viscosity $\rightarrow$

- strong coupling between dilatational modes and internal degrees of freedom

- Production of large number of soft partons

- Screening of color charge of pre-existing quarks and gluons

\section{- Soft statistical hadronization}

- Decrease in $\left\langle p_{T}\right\rangle$ and increase of $M$ due to rapid increase in entropy and associated quenching of transverse hydro expansion

(Observed effect for ${ }^{3} \mathrm{He}$ ) 48 


\section{A possible collective mode of excitation}

Using 3-particle correlation to discriminate different physical mechanisms.

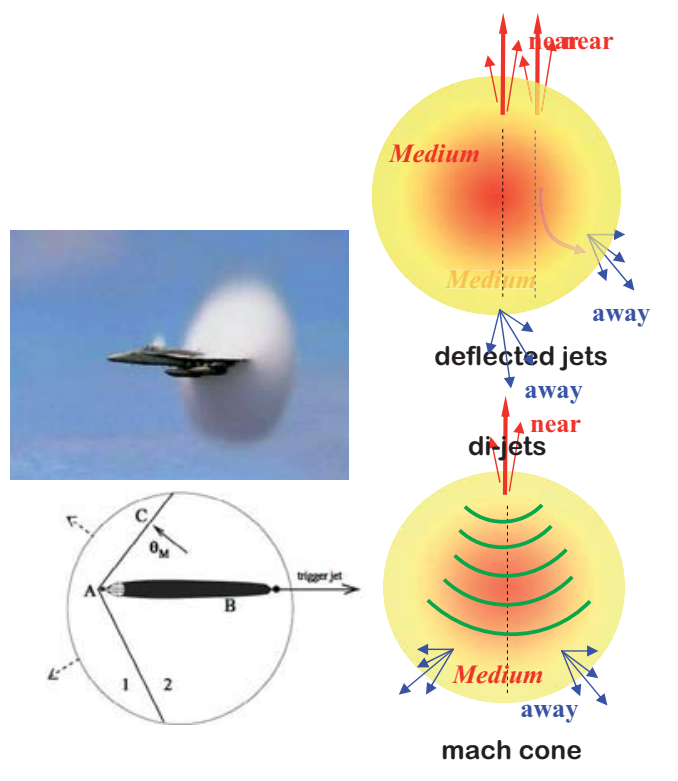

TJH: JINR Heavy Ion Summer Schoo
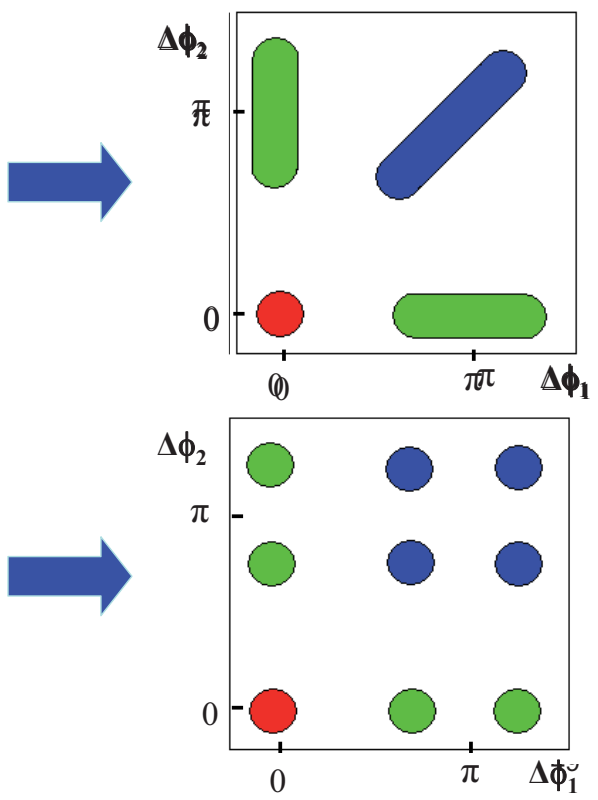

July 14-26 Dubna, Russia

\section{The experimental evidence}

Indications of Conical Emission of Charged Hadrons at RHIC arXiv:0805.0622v1 [nucl-ex] 6 May 2008

..... Distinct peaks at $\theta=1.38 \pm 0.02$ (stat) \pm 0.06 (syst.) Irom $\pi$ are observed on the away side in central $\mathrm{Au}+\mathrm{Au}$ collisions, with correlated hadron pairs far apart, symmetric about $\pi$, as well as close together. These structures are evidence of conical emission of hadrons correlated with high $p_{\perp}$ particles.
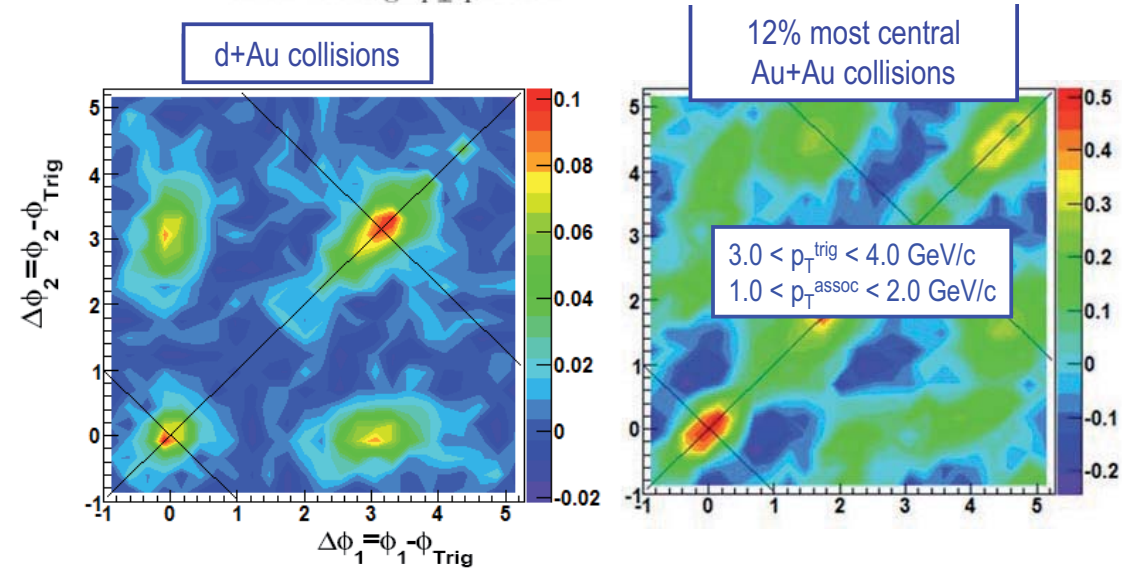


\section{Future tools}

\section{Deconfinement and color screening?}

- Classic proposal: quarkonium suppression by color screening.

- Lattice QCD calculations tell us the world is more complicated than we thought! Quarkonium resonances should persist above $T_{c}$.

- Hierarchy of melting:

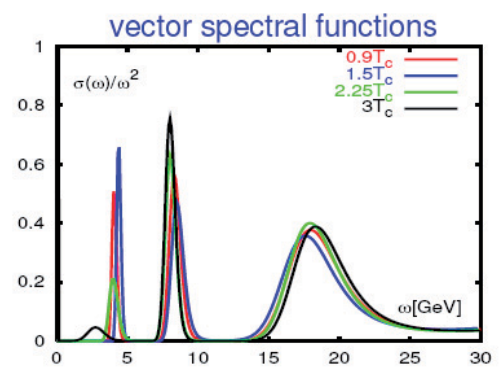

\begin{tabular}{|c||c|c|c||c|c|c|c|c|}
\hline State & $J / \psi(1 S)$ & $\chi_{c}(1 P)$ & $\psi^{\prime}(2 S)$ & $\Upsilon(1 S)$ & $\chi_{b}(1 P)$ & $\Upsilon(2 S)$ & $\chi_{b}(2 P)$ & $\Upsilon(3 S)$ \\
\hline $\mathrm{T}_{d} / \mathbf{T}_{c}$ & 2.10 & 1.16 & 1.12 & $>4.10$ & $<\mathbf{1 . 7 6}$ & 1.60 & 1.19 & 1.17 \\
\hline
\end{tabular}

- Also recombination: $\quad c+\bar{c} \rightarrow J / \Psi$ 


\section{Current status}
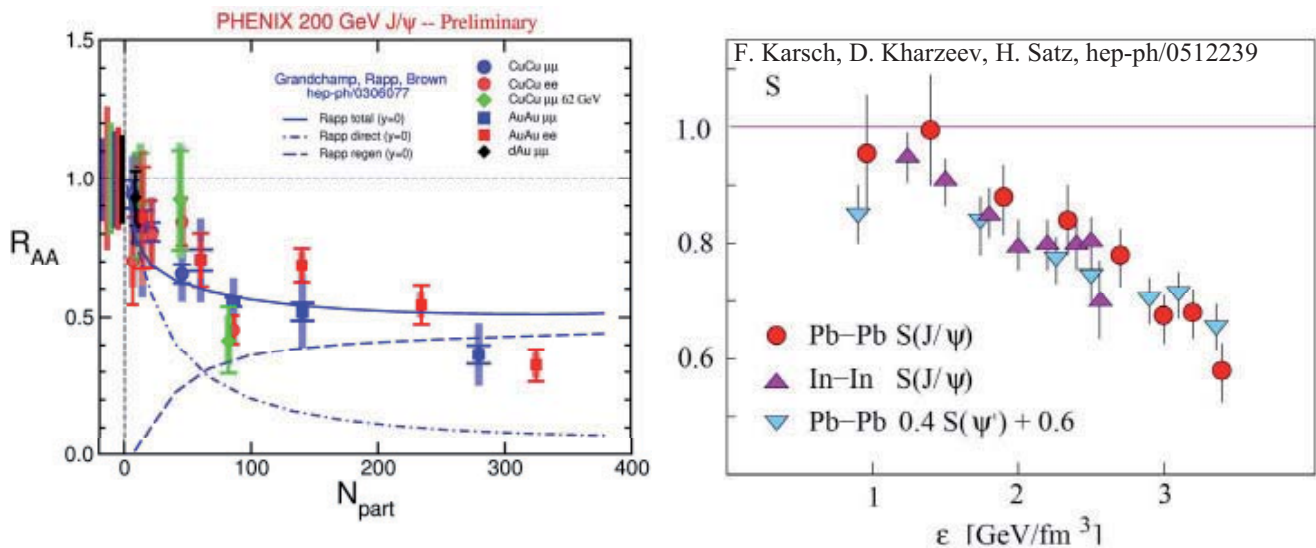

- Suppression + regeneration describes PHENIX results well

- Sequential melting also works if you assume the $\mathrm{J} / \Psi$ doesn't melt

\section{How to discriminate?}

- Compare model predictions to measurements of:

- $J / \Psi$ spectrum modifications vs. rapidity and beam energy

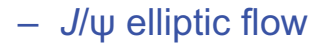

- Need $\psi^{\prime}$ and $X_{c}$ measurements, both as inputs to the model calculations and to provide direct evidence for melting

- Need bottomonium (separated 1s,2s,3s), where the expected effects are quite different from charmonium

- These measurements require upgraded detector capabilities and higher "RHIC II" luminosity 


\section{A future test of color screening in the plasma: Bottomonium $(Y)$}

First $\Upsilon$ measurement
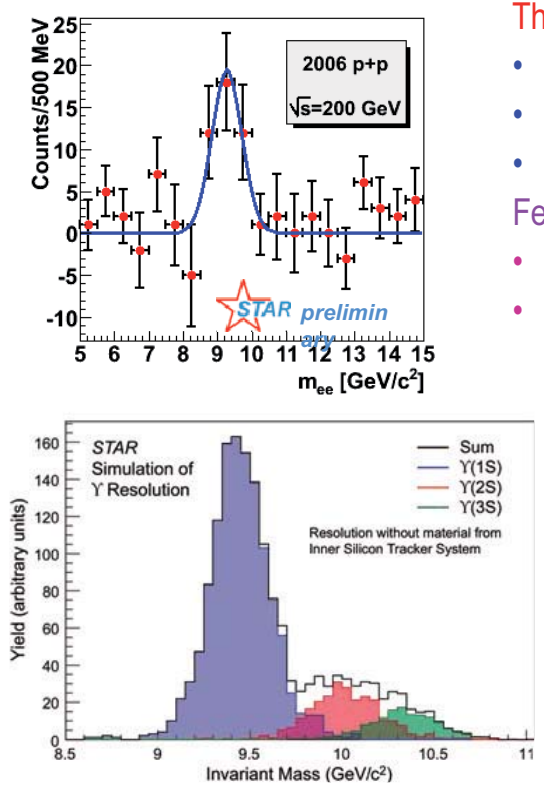

The $\Upsilon, \Upsilon^{\prime}, \Upsilon^{\prime \prime}$ should behave differently than the $\mathrm{J} / \Psi$

- $\Upsilon(1 \mathrm{~S})$ no melting at RHIC $\Rightarrow$ standard candle

- $\Upsilon(2 S)$ likely to melt at RHIC (analog $\mathrm{J} / \psi$ )

- $\quad \Upsilon(3 S)$ melts at RHIC (analog $\psi^{\prime}$ )

\section{Features}

- co-mover absorption negligible

- recombination negligible at $\mathrm{RHIC}$

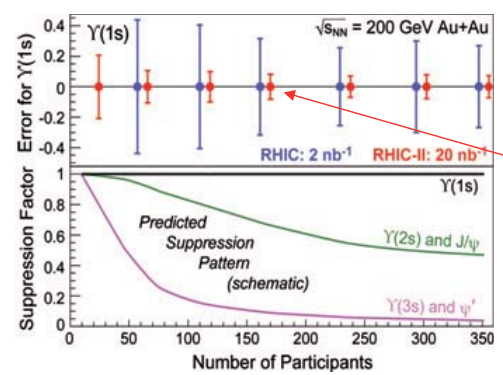

$\Upsilon \rightarrow \mathrm{e}^{+} \mathrm{e}^{-}$

T. Ullrich et al

Statistical precision Expected with RHIC ॥ luminosity

TJH: JINR Heavy Ion Summer School

July 14-26 Dubna, Russia

\section{RHIC-II Science Goals: Quantifying Properties of the Perfect Liquid}

Enhanced luminosity (by 2012) + detector upgrades will enable rare probe studies of quarkonium (qqbar systems) yield \& flow, sensitive to color screen-ing (deconfinement) and parton equilibration/coalescence in the QGP.
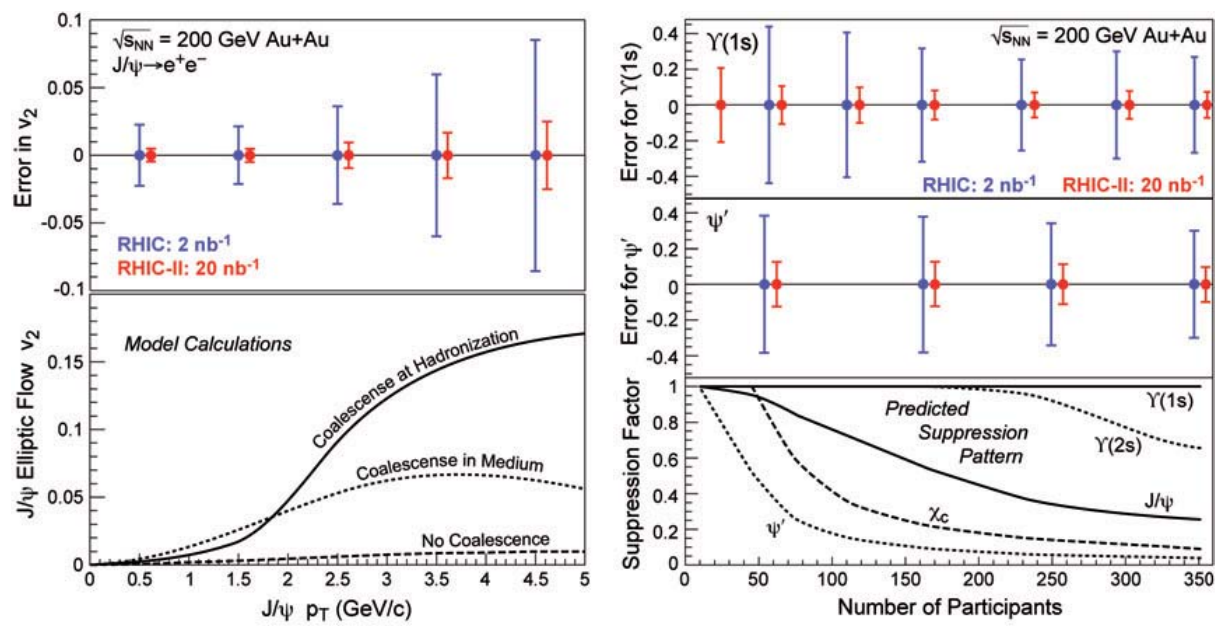


\section{$\gamma$-Jet: Golden Probe of QCD Energy Loss}
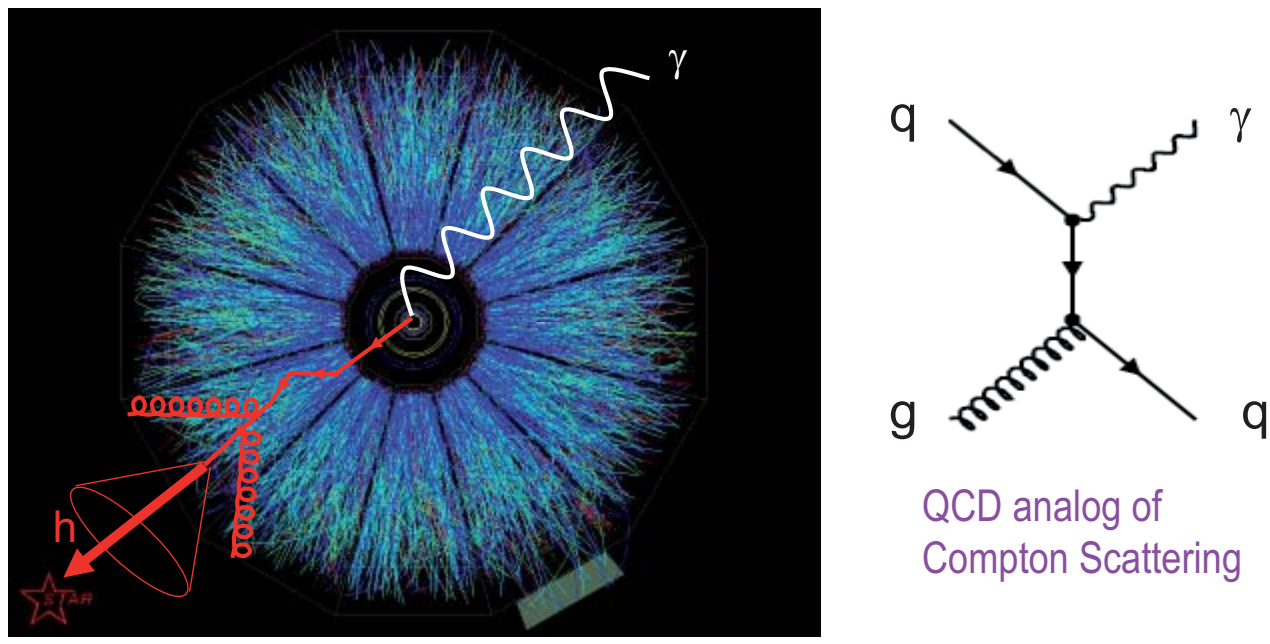

QCD analog of

Compton Scattering

$\square \quad \gamma$ emerges unscathed from the medium

- This probe is valuable for comparison with di-hadron correlations

- It provides fully reconstructed kinematics: measure real fragmentation function $D(z)$

TJH: JINR Heavy lon Summer School

July 14-26 Dubna, Russia

\section{The next step for one of the detectors}

Full prototype TPC sector now in operation and working flawlessly

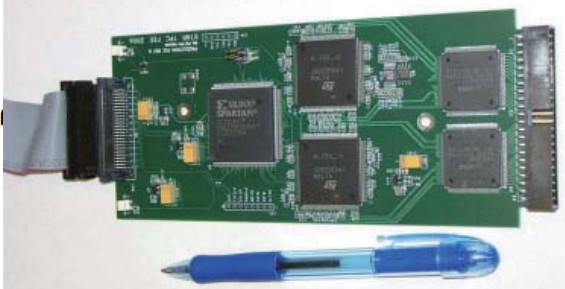

STAR DAQ1000 FEE

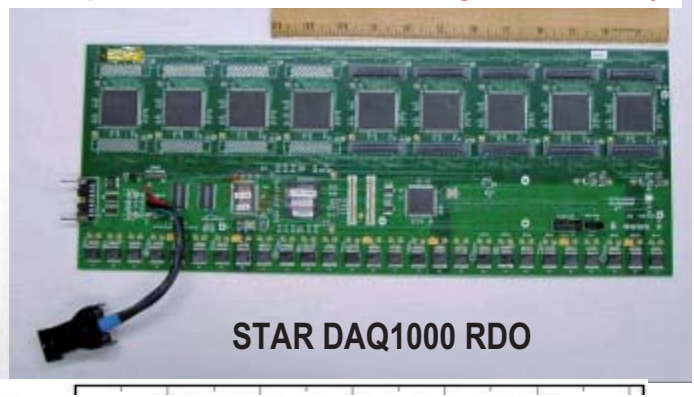

Collaboration Plan:

Increase of DAQ rate to1000 times design by Run 9 leveraging CERN/ALICE Altro chip development (thank you)

Construction of HFT in time for full operation in Run 12 (Fall 2011) 
Five "final item" mrpc trays in place and in the STAR data stream
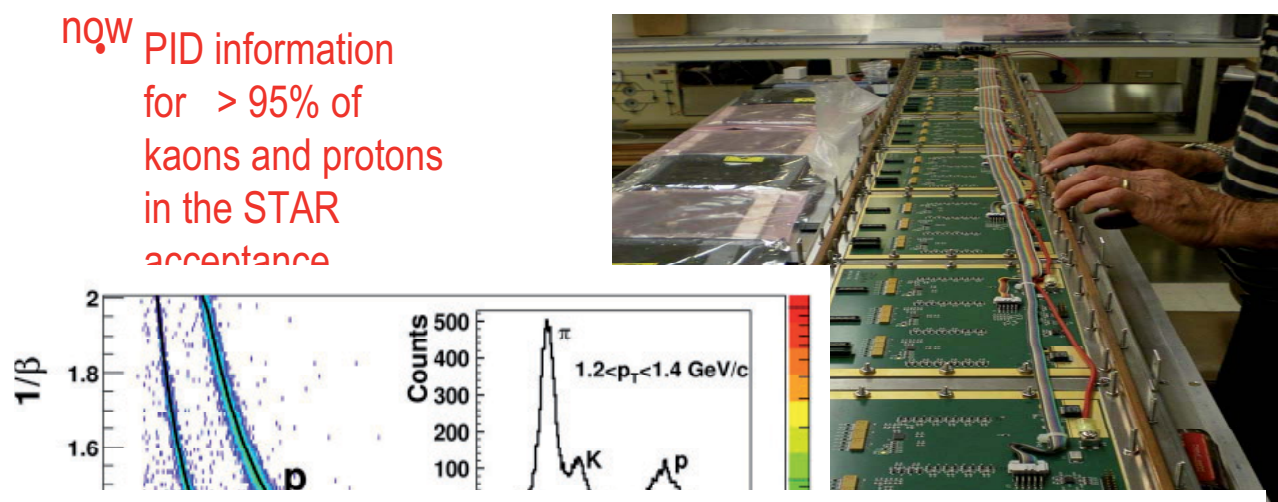

Collaboration plan

120 trays of MRPC modules which leverage

MRPC development at CERN (Crispin Williams et al)

Development of HPTDC Chip

MRPC tech transfer and construction (contributed) in China

Fully complete in time for run 10 (fall 2009)

Future di-lepton program to study in-medium effects
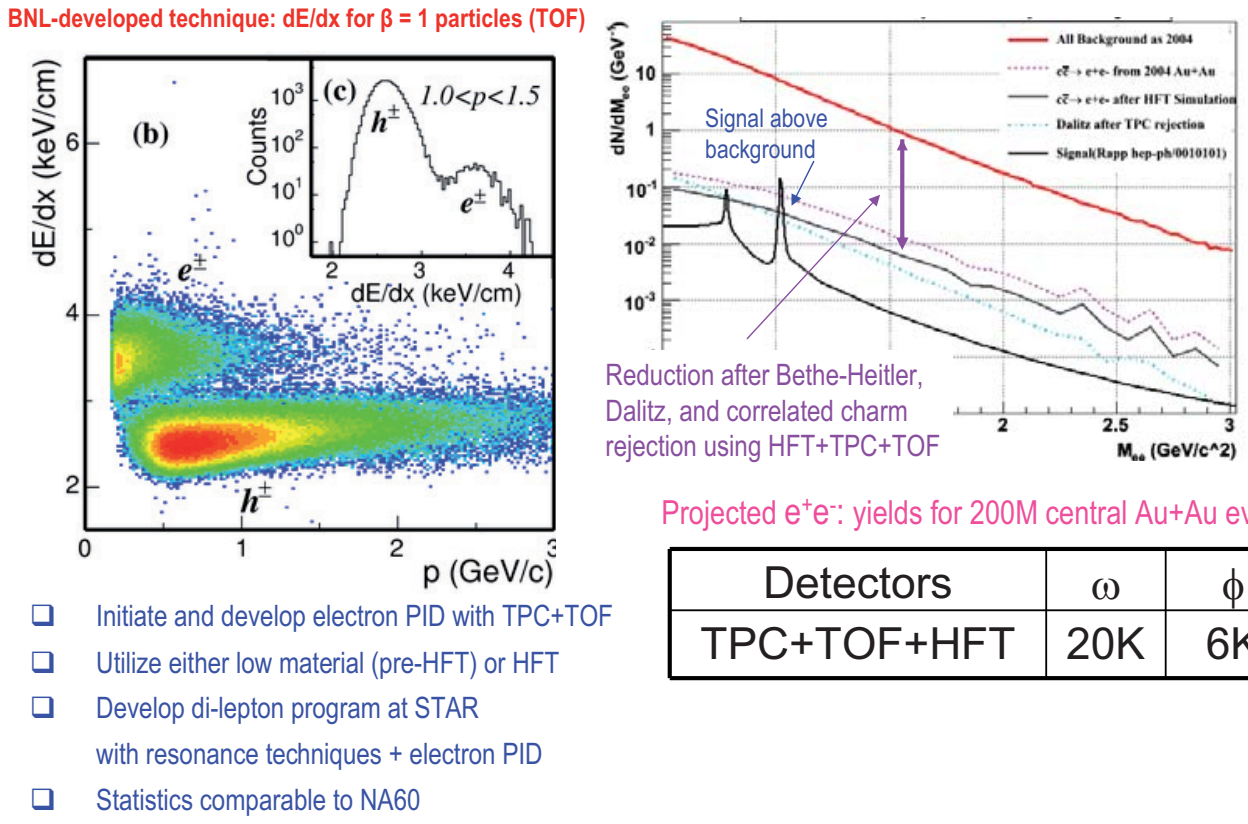

Projected $\mathrm{e}^{+} \mathrm{e}^{-:}$: yields for $200 \mathrm{M}$ central $\mathrm{Au}+\mathrm{Au}$ events

\begin{tabular}{|c|c|c|}
\hline Detectors & $\omega$ & $\phi$ \\
\hline TPC+TOF+HFT & $20 \mathrm{~K}$ & $6 \mathrm{~K}$ \\
\hline
\end{tabular}

Develop di-lepton program at STAR with resonance techniques + electron PID

Statistics comparable to NA60 


\section{A Long Term (Evolving) Strategic View for RHIC}

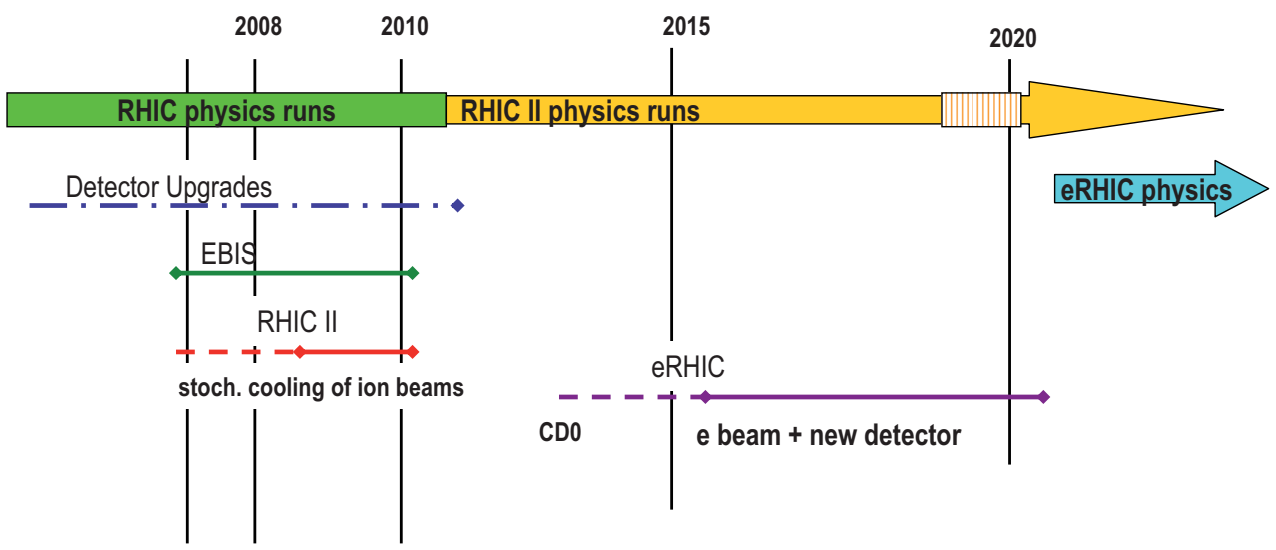

\section{Legend:}

- - - - - R\&D

$\longrightarrow$ Construction

$-\cdot-\cdot \rightarrow$ Multiple small projects

CDO: DOE Critical Decision, mission need

TJH: JINR Heavy lon Summer School

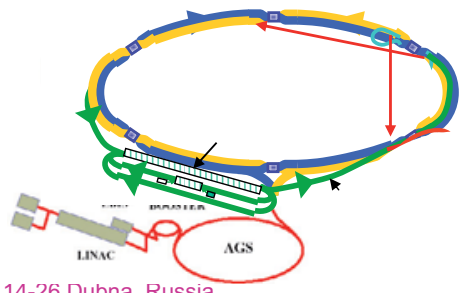

July 14-26 Dubna, Russia

And then, there is the question of the initial conditions 
What are the initial-state parton distribution functions and how they effect the time it takes to thermalize

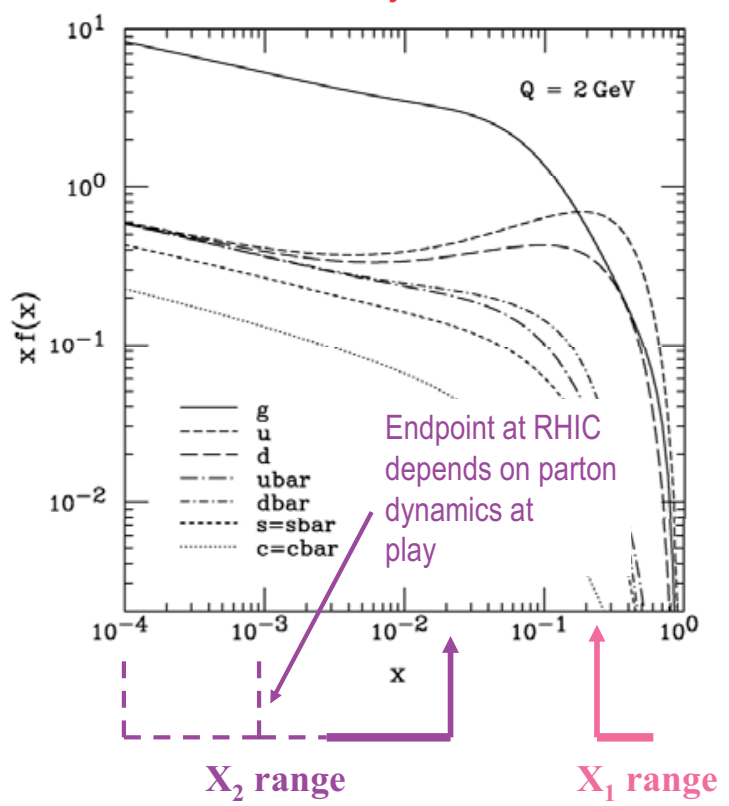

Measurements needed at high rapidity to set the dominant parton type:

Projectile $\left(x_{1} \sim 1\right)$ mostly valence quarks.

Target $\left(x_{2}<0.01\right)$ mainly gluons.

Sensitive to $\mathrm{x}_{\mathrm{g}} \sim 10^{-3}$ in $\mathrm{PQCD}$ picture Sensitive to $\mathrm{x}_{\mathrm{g}} \sim 10^{-4}$ in CGC picture

\section{- Question}

- At low-x in the previous plot, the gluon distribution continues to grow exponentially

- But, it can not grow indefinitely without bound - What happens at when $\mathrm{x}$ becomes very small ? 
Attempt at a semi-classical, effective field approach: conceptual expectation for a Color Glass Condensate

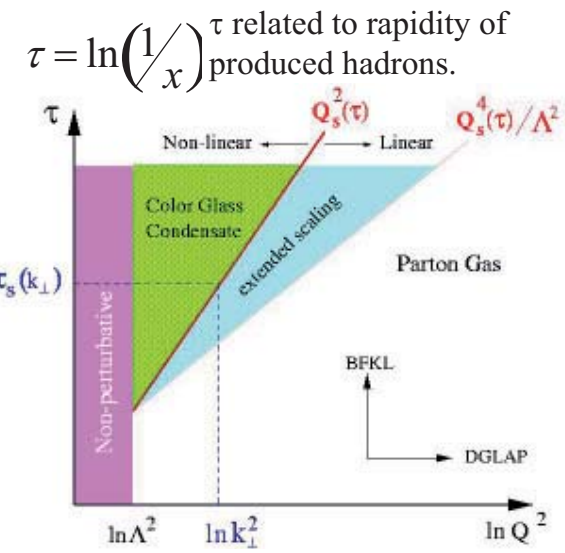

Iancu and Venugopalan, hep-ph/0303204

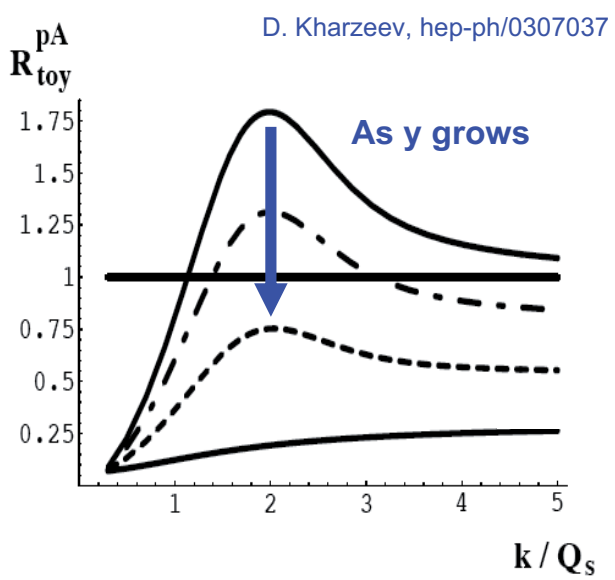

Is there evidence for gluon saturation at RHIC energies?

\section{Forward particle production in $\mathrm{d}+\mathrm{Au}$ collisions}
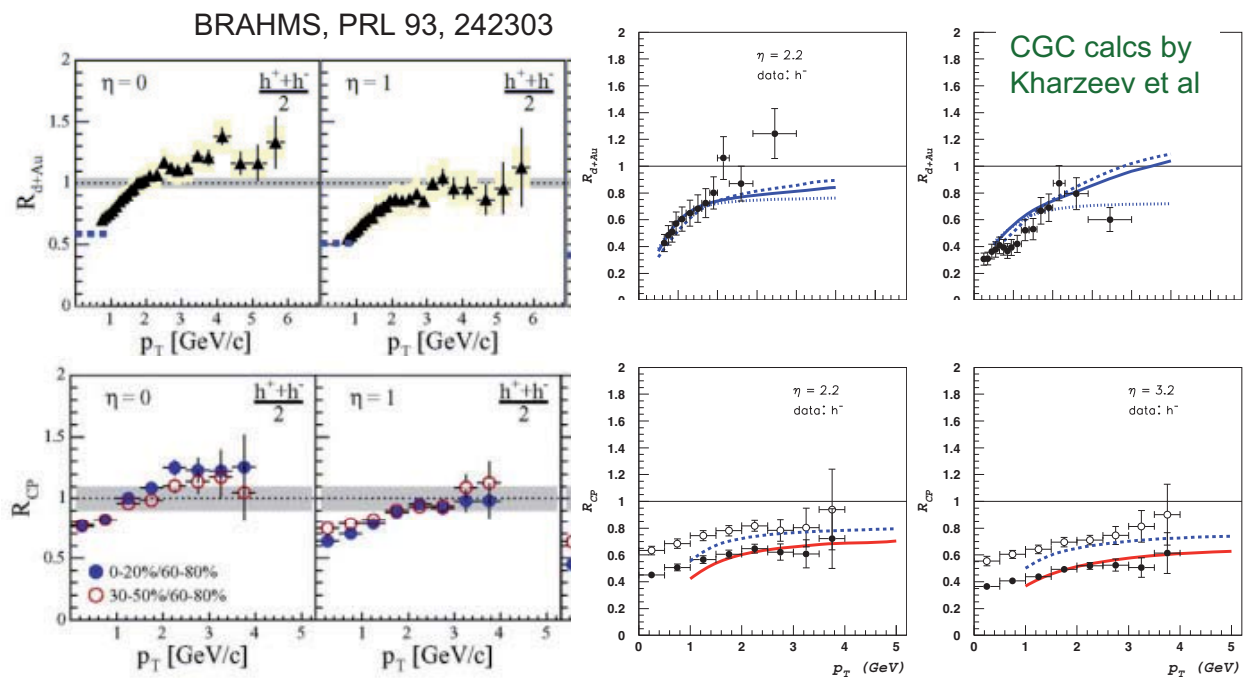

- Sizable suppression of charged hadron yield in forward $d+A u$

- Evidence for a saturated gluon field in the Au nucleus?

- Several other mechanisms have also been proposed 


\section{STAR Nuclear Modification Factor at high rapidity}

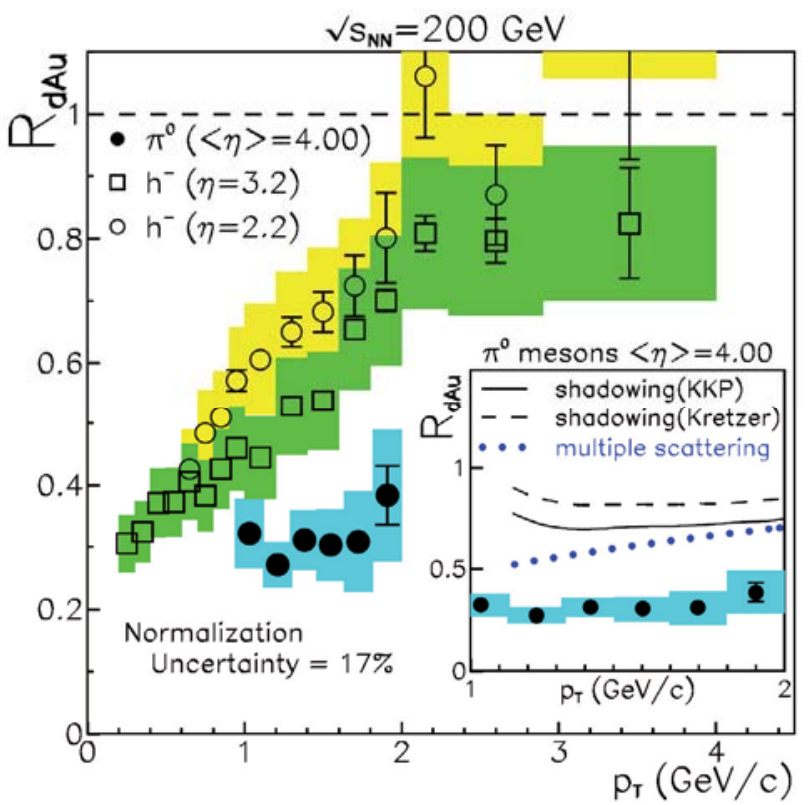

$R_{\mathrm{dAu}}^{Y}=\frac{\sigma_{\text {inel }}^{p p}}{\left\langle N_{\text {bin }}\right\rangle \sigma_{\text {hadr }}^{d A u}} \frac{E d^{3} \sigma / d p^{3}(d+A u \rightarrow Y+X)}{E d^{3} \sigma / d p^{3}(p+p \rightarrow Y+X)}$

BRAHMS published result consistent with new STAR once an isospin suppression of $h$ - in $p+p$ is taken into account (factor of $2 / 3$ ).

Calculations that do not include mod. of Au wave function cannot reproduce data.

\section{Correlations will provide a more sensitive probe}

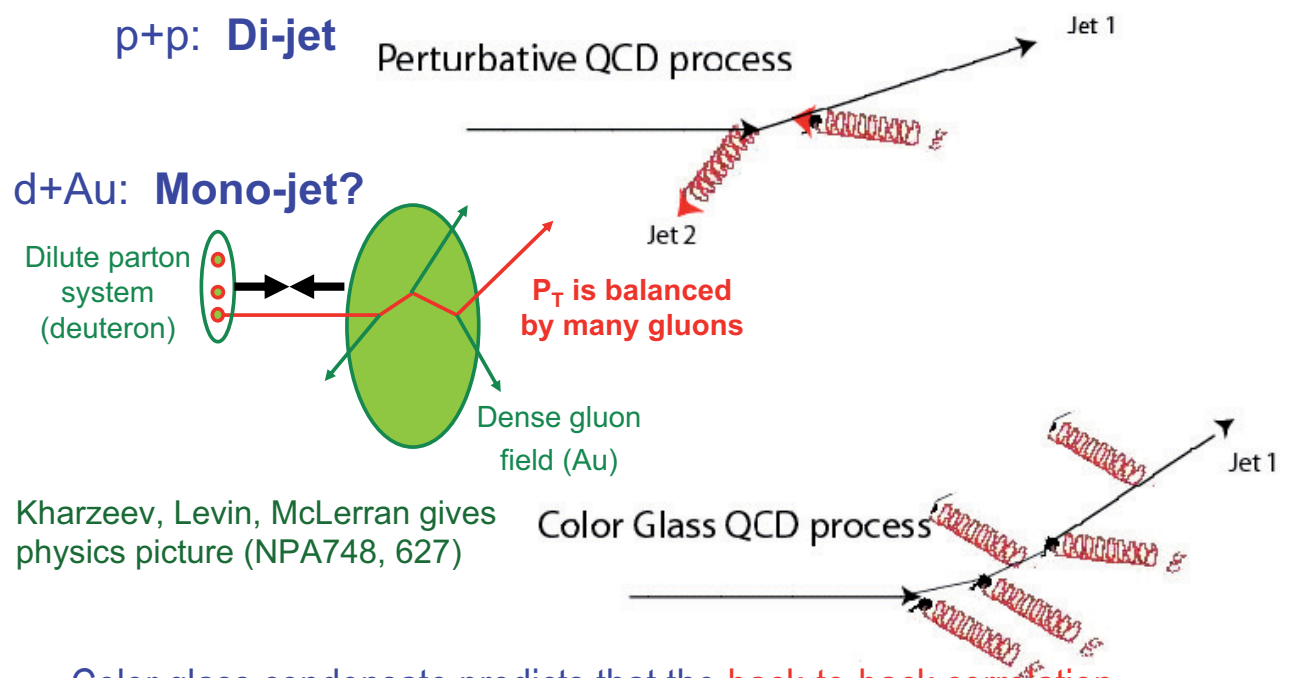

Color glass condensate predicts that the back-to-back correfation from $p+p$ should be suppressed 
An initial glimpse: correlations in $d+A u$

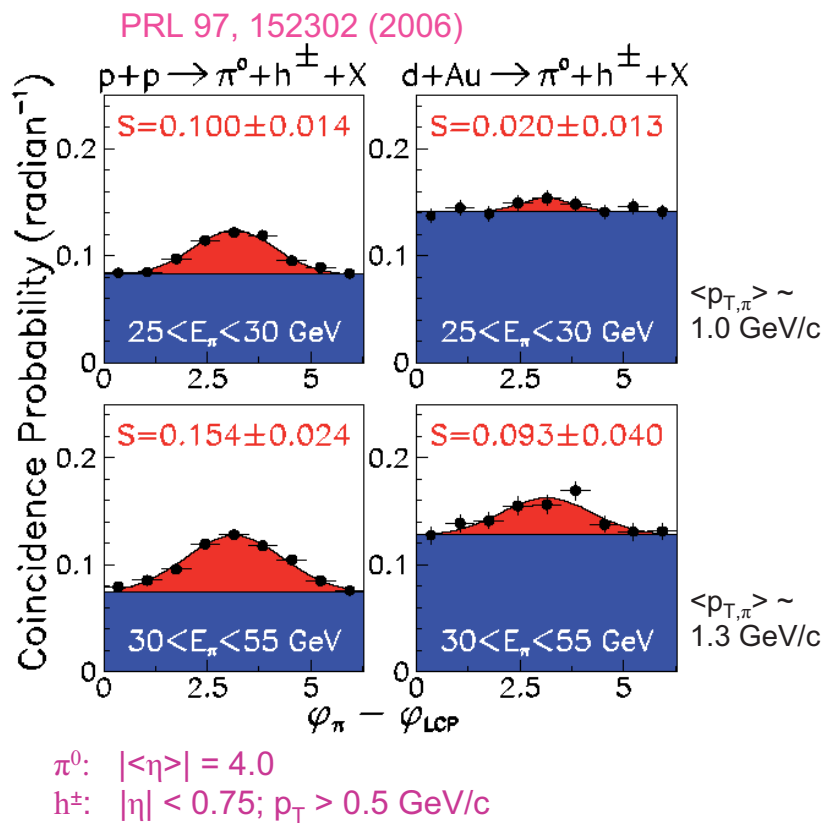

- are suppressed at small $\left\langle\mathrm{x}_{\mathrm{F}}\right\rangle$ and $\left\langle\mathrm{p}_{\mathrm{T}, \pi}\right\rangle$ consistent with
CGC picture

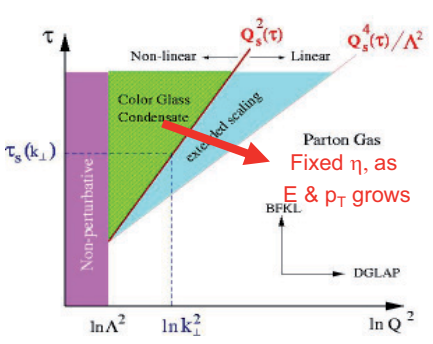

- are similar in $\mathrm{d}+\mathrm{Au}$ and $p+p$ at larger $\left\langle x_{F}\right\rangle$ and $\left\langle\mathrm{p}_{\mathrm{T}, \pi}\right\rangle$

As expected by HIJING

TJH: JINR Heavy lon Summer School

July 14-26 Dubna, Russia

L $_{\text {TAR }} p+p$ and $d+A u \rightarrow \pi^{0}+\pi^{0}+X$ correlations with forward $\pi^{0}$
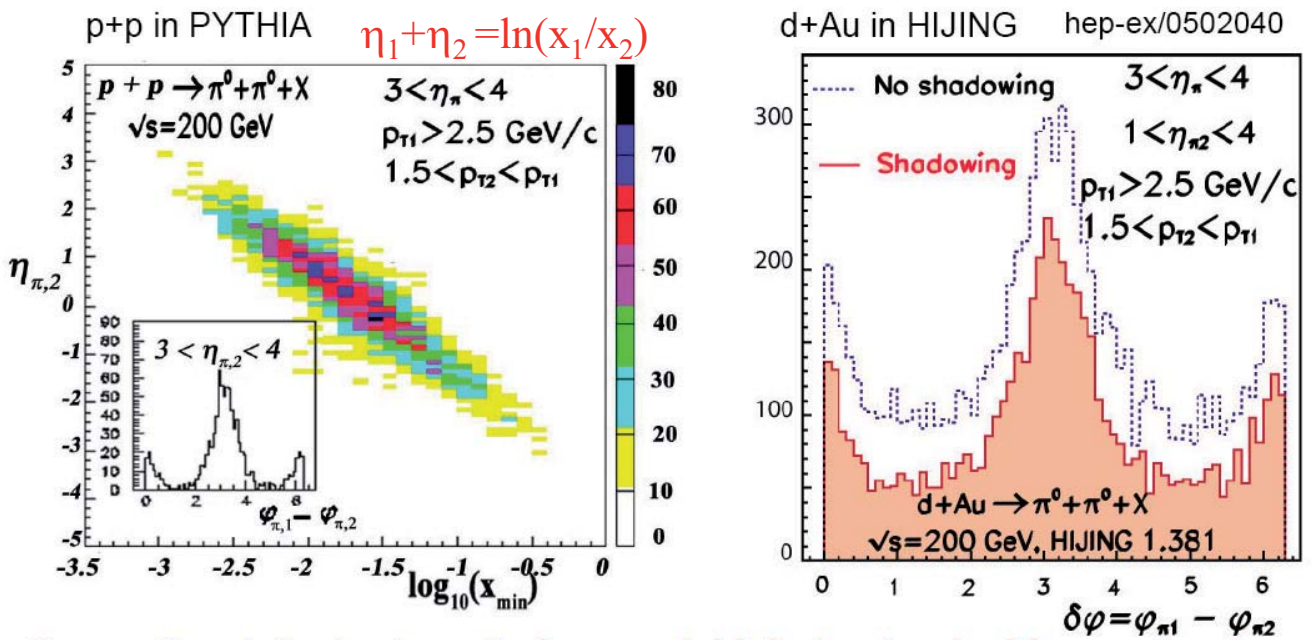

Conventional shadowing will change yield, but not coincidence structure. Coherent effects such as CGC evolution will change the structure. Sensitive to $x_{g} \sim 10^{-3}$ in pQCD scenario; few $\times 10^{-4}$ in CGC scenario. 


\section{Ultimately, to study the low- $x$ gluon distribution in heavy nuclei properly, a new Electron-lon Collider (EIC) is needed (BNL + TJNAF)}

\section{What has been found: $3^{+}$new discoveries}

- Enormous collective motion of the medium, consistent with near-zero viscosity hydrodynamic behavior

- Very fast thermalization

- A "perfect liquid"

- Jet quenching in the dense matter

- Densities up to 100 times cold nuclear matter and 15 times the critical density from lattice calculations

- Anomalous production of baryons relative to mesons

- Strongly enhanced yields of baryons relative to mesons

- Scaling of yields and collective motion with the number of valence quarks

- Hadrons form by constituent quark coalescence

- Indications of gluon saturation in heavy nuclei

- Relatively low multiplicities in Au+Au collisions

- Suppressed particle production in $d+A u$ collisions 


\section{New scientific questions}

- What is the mechanism of the unexpectedly fast thermal equilibration?

- What is the initial temperature and thermal evolution of the produced matter?

- What is the energy density and equation of state of the medium?

- What is the viscosity of the produced matter?

- Is there direct evidence for deconfinement, color screening, and a partonic nature of the hot, dense medium? What is the screening length?

- Can we directly observe a QCD phase transition? Where is the QCD critical point?

- Is chiral symmetry restored, as predicted by QCD?

- How does the new form of matter hadronize at the phase transition?

\section{These are the topics of RHIC II......}

First results on high- $p_{T}$ di-hadron, $\gamma$-hadron correlations

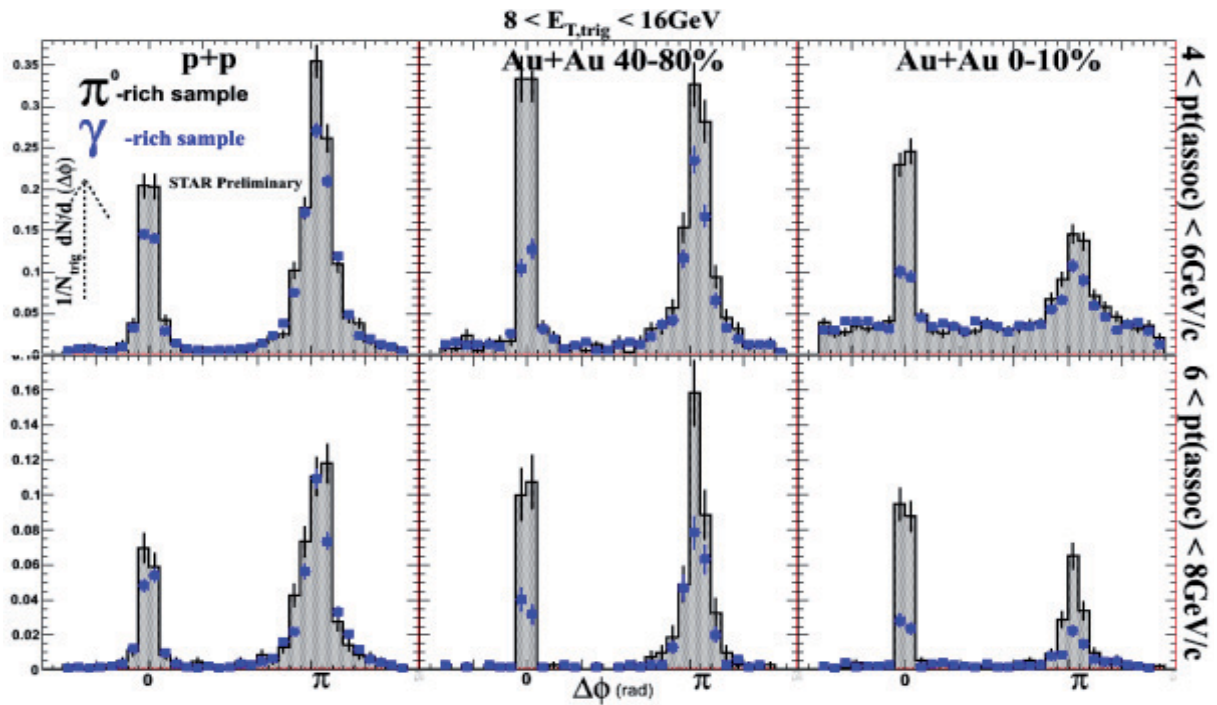

First steps to precision study with high luminosity at RHIC 


\section{Stochastic Cooling at RHIC}

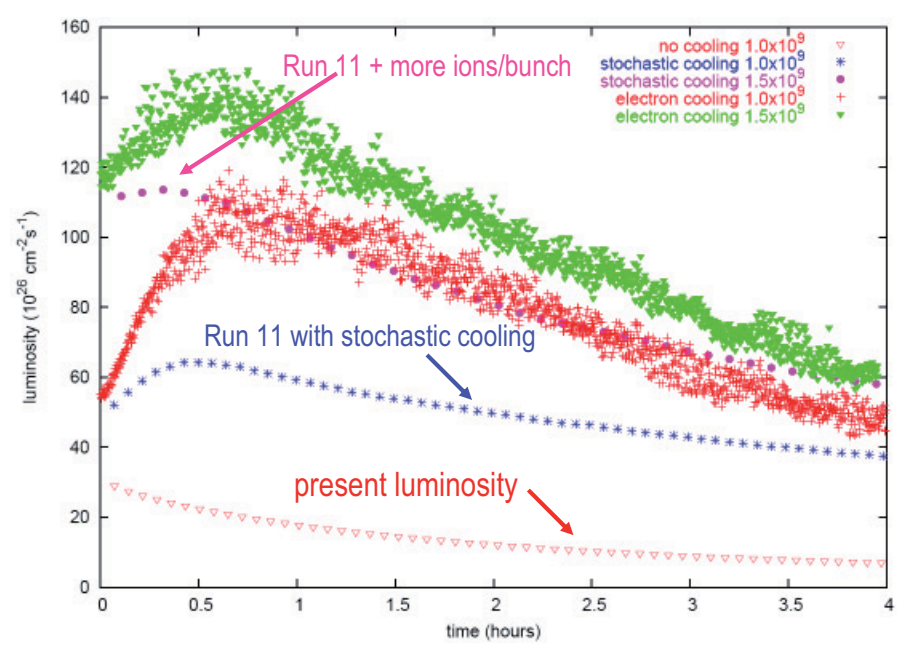

Figure 2. Simulations of the luminosity vs. time during a 4-hour store for $V_{s} N N=200 \mathrm{GeV} A u+A u$ collisions, with and without beam cooling and for two different assumed initial bunch intensities. The performance ${ }_{75}$ of electron-cooled vs. stochastically cooled beams is compared.

TJH: JINR Heavy Ion Summer School

July 14-26 Dubna, Russia

Relevance of the answer to the QGP: $v_{2}$ and the hydro limitGlauber vs Color Glass Condensate

Hirano et al, PLB 636, 299
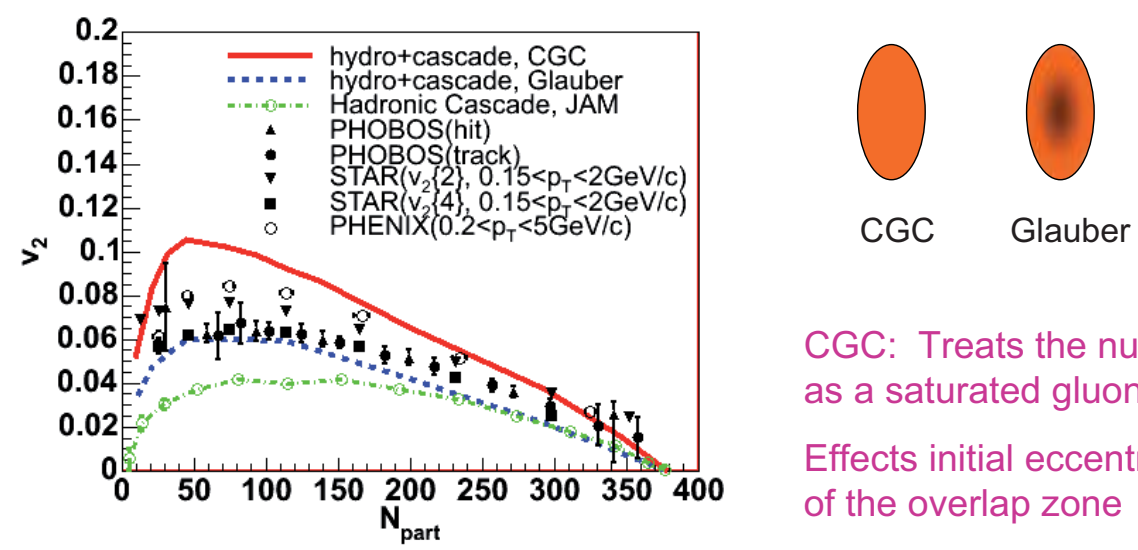

CGC: Treats the nucleus as a saturated gluon field Effects initial eccentricity of the overlap zone

- Do we have Glauber matter distribution + perfect liquid, or Color Glass Condensate distribution + viscous matter?

- Understanding the initial state is crucial to understand what we are seeing in the final state 


\section{In fact there is a strong coupling to understanding the story at mid-rapidity:}

Answers are (urgenetly) needed to:

Understand the initial state conditions for relativistic heavy nuclei at RHIC and confirm our understanding of multiplicities and rapidity dependence

Understand how thermalization appears to be established so quickly at RHIC

Understand whether we have really reached the hydro limit for $\mathrm{v}_{2}$

Understand mid-rapidity particle production at the LHC in the future

\section{Complementarity of RHIC II and LHC}

- Far more heavy quarks per collision at LHC:

- Head-on Au+Au collision at RHIC: $\mathrm{N}_{\mathrm{cc}} \sim 10 \quad \mathrm{~N}_{\mathrm{bb}} \sim 0.05$

- Head-on Pb+Pb collision at LHC: $\quad \mathrm{N}_{\mathrm{cc}} \sim 115 \quad \mathrm{~N}_{\mathrm{bb}} \sim 5$

- Far more collisions per year at RHIC II

- Detected quarkonium per year will be comparable at RHIC II (full energy) and LHC

- But:

- For charmonium: mixture of effects at full energy RHIC II, can turn off recombination with longer runs at lower energy; recombination at LHC

- For bottomonium: pure suppression at full energy RHIC II; mixture of effects at LHC

- Both RHIC II and LHC will be essential to gain maximal information from either 


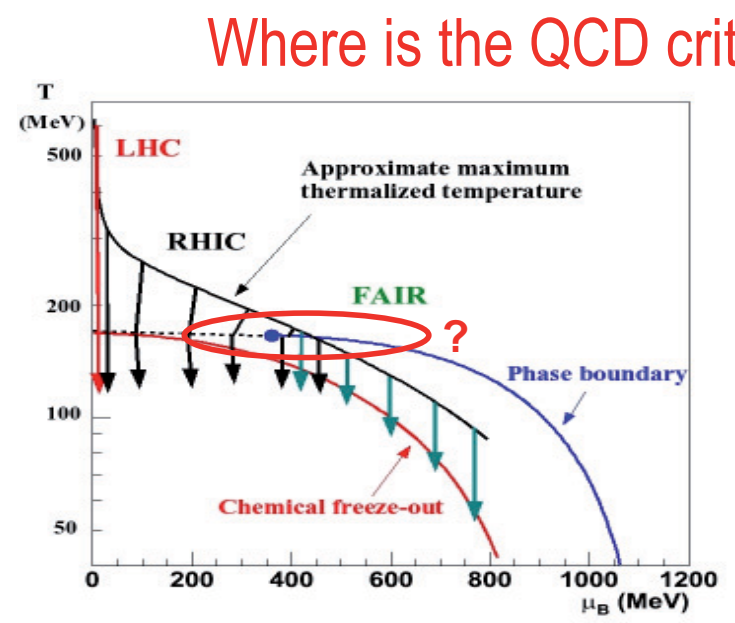

- The "landmark" on the QCD phase diagram!

- Lattice calculations: between $\mu_{\mathrm{B}}$ of $<\sim 200$ and $>\sim 700 \mathrm{MeV}$.

- $\mathrm{RHIC}$ can find it if $\mu_{\mathrm{B}}<500 \mathrm{MeV}$

- Need detailed study of many different collision energies

- Significant advantage of RHIC: with collider detectors, most systematic effects are constant with beam energy

- Low energy electron cooling would greatly increase the luminosity

\section{Evidence for thermalization in sQGP}

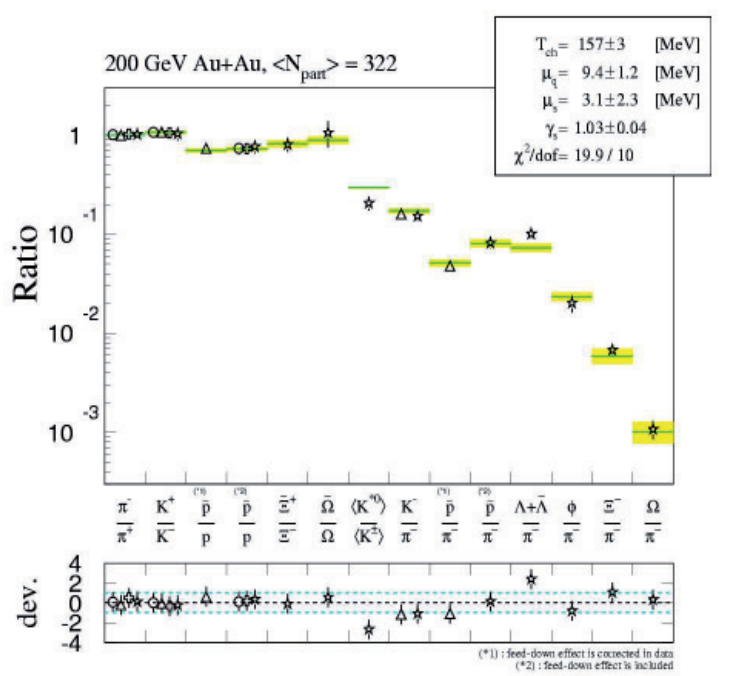

$\mathrm{p}_{\mathrm{T}}$-integrated particle yield ratios in central Au+Au collisions consistent with Grand Canonical Stat. distribution @ $\mathrm{T}_{\mathrm{ch}}=(160 \pm 10) \mathrm{MeV}$, $\mu_{\mathrm{B}} \approx 25 \mathrm{MeV}$, across $\mathrm{u}, \mathrm{d}$ and s quark sectors.

Inferred Temp. consistent with $\mathrm{T}_{\text {crit }}$ $($ LQCD) $\Rightarrow$ phase transition

- Particle composition consistent with chemical equilibrium among hadrons

- Largest deviation $\left(\mathrm{K}^{*}\right)$ arises from its short lifetime in the hadron gas phase 


\section{Particle multiplicity vs. pseudorapidity}

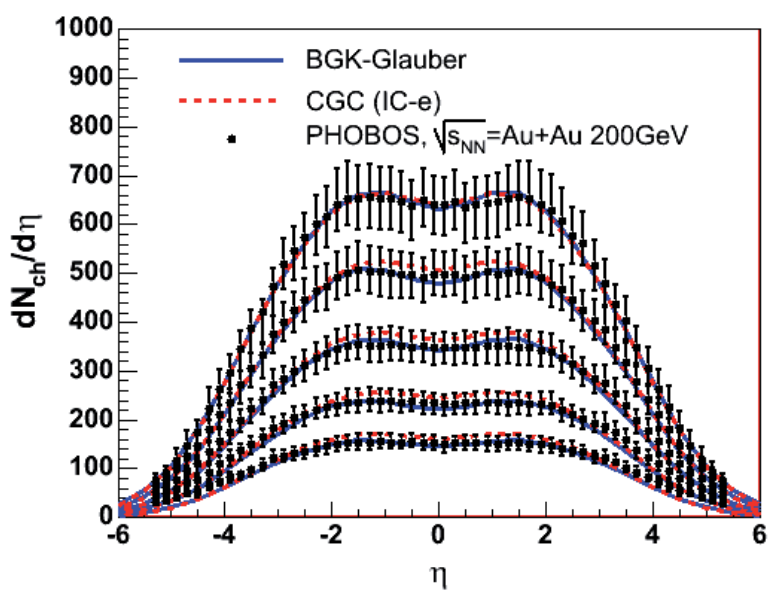

- Multiplicities well described by Color Glass Condensate model

- Evidence for saturated gluon fields in the Au nucleus?

In principle, there is a lot of information needed to understand High $\mathrm{p}_{\mathrm{T}}$ Particle Production in A+A Collisions $\frac{d N_{A B}^{h}}{d y d^{2} p_{T}}=K \sum_{a b c d} \int d x_{a} d x_{b} \int d^{2} \mathbf{k}_{a} d^{2} \mathbf{k}_{b}$

(According to $\mathrm{pQCD}$...)

$\times f_{a / A}\left(x_{a}, Q^{2}\right) f_{b / B}\left(x_{b}, Q^{2}\right) \quad$ Parton Distribution Functions

$\times g\left(\mathbf{k}_{a}\right) g\left(\mathbf{k}_{b}\right) \quad$ Intrinsic $\mathbf{k}_{\mathrm{T}}$, Cronin Effect

$\times S_{A}\left(x_{a}, Q_{a}^{2}\right) S_{B}\left(x_{b}, Q_{b}^{2}\right) \quad$ Shadowing, EMC Effect

$\times \frac{d \sigma}{d \hat{t}}(a b \rightarrow c d)$

Hard-scattering cross-section

$\times \int_{0}^{1} d \varepsilon P(\varepsilon) \frac{z_{c}^{*}}{z_{c}}$

Partonic Energy Loss

$\underline{D_{h / c}^{0}\left(z_{c}^{*}, Q_{c}^{2}\right)}$ $\pi z_{c}$

\section{Fragmentation Function}

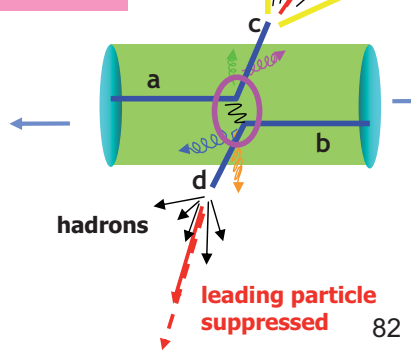


But using our calibrated probe we can try something simpler to start

Looking along the beam line
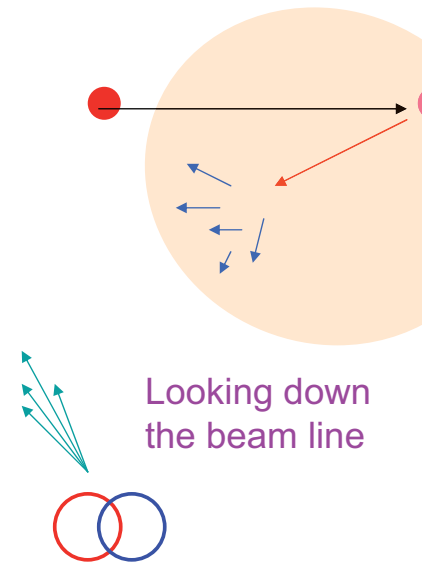

If some new form of dense, opaque matter is formed, we might see the effects on our "calibrated" probe (back-to-back correlation)

\section{Gas of weakly/strongly interacting Li atoms}

M. Gehm et al, Science 298, 2179

- excite Feshbach resonance
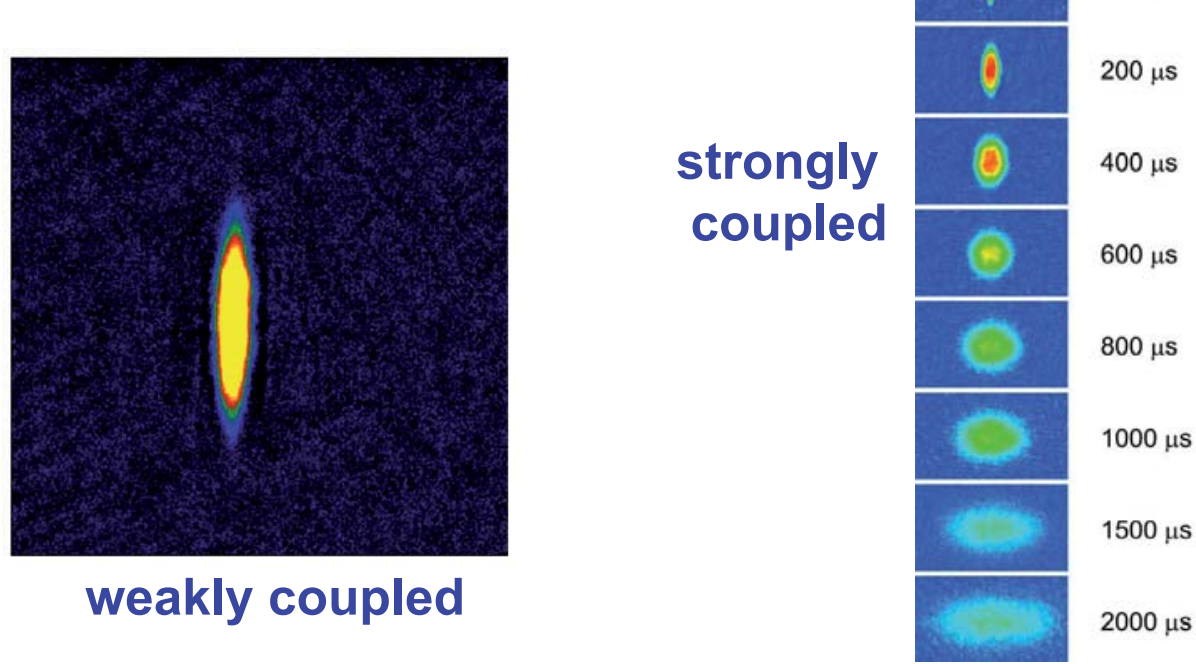


\section{RHIC / RHIC-II / LHC-HI EIC $\Rightarrow$ Laboratory Exploration of Condensed Strongly Interacting Matter}

Profound questions: What are the unique quantum many-body manifestations of a non-Abelian gauge theory? Are there lessons for other fundamental theories? How do we pump/probe partonic matter in $10^{-23} \mathrm{~s}$ ?

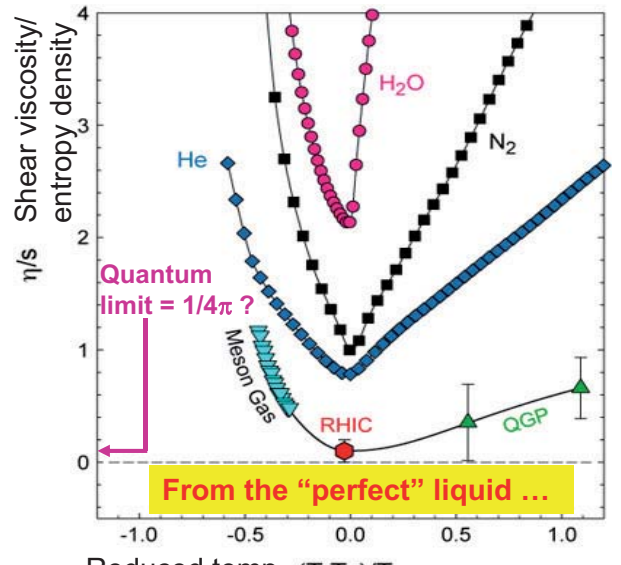

Reduced temp. $\left(\mathrm{T}-\mathrm{T}_{0}\right) / \mathrm{T}_{0}$

RHIC, RHIC-II, perhaps LHC probe very strong coupling limit: $L Q C D \Rightarrow$ quantitative theory for static properties; AdS/CFT $\Rightarrow$ qualitative insight + gravity connection.

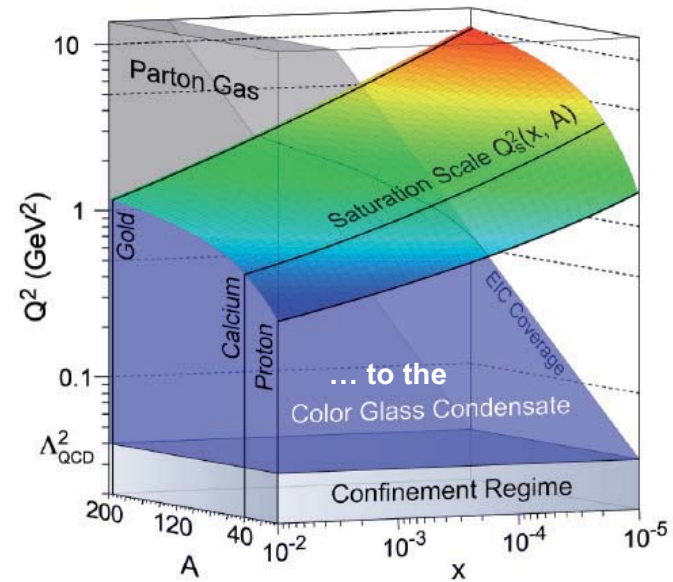

EIC probes weak coupling regime with gauge boson occupancy $\gg 1 \Rightarrow$ intense semiclassical (gluon) force field at the heart of matter.

\section{Collective motion: "elliptic flow"}
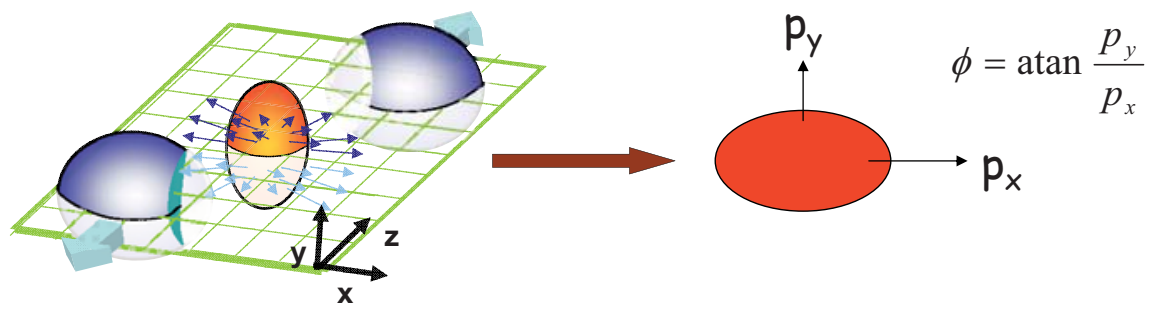

$$
\varepsilon=\frac{\left\langle y^{2}\right\rangle-\left\langle x^{2}\right\rangle}{\left\langle y^{2}\right\rangle+\left\langle x^{2}\right\rangle}
$$

Initial coordinate-space anisotropy

$$
v_{2}=\left\langle\frac{p_{x}{ }^{2}-p_{y}{ }^{2}}{p_{x}{ }^{2}+p_{y}{ }^{2}}\right\rangle
$$

Final momentum-space anisotropy

$$
\begin{aligned}
& \frac{d N}{d \phi} \propto 1+2 v_{2} \cos \left[2\left(\phi-\Psi_{R}\right)\right]+2 v_{4} \cos \left[4\left(\phi-\Psi_{R}\right)\right]+\ldots \\
& \text { Anisotropy self-quenches, so } \\
& \text { Ellintin torm } \\
& v_{n} \text { is sensitive to earlv times }
\end{aligned}
$$

Elliptic flow establishes there is strongly interacting matter at $\mathrm{t} \sim 0$ 